\title{
On Sectional Paths in a Category of Complexes of Fixed Size
}

\section{Claudia Chaio, Isabel Pratti \& María José Souto-Salorio}

Algebras and Representation Theory

\section{ISSN 1386-923X}

Algebr Represent Theor

DOI 10.1007/s10468-016-9643-2

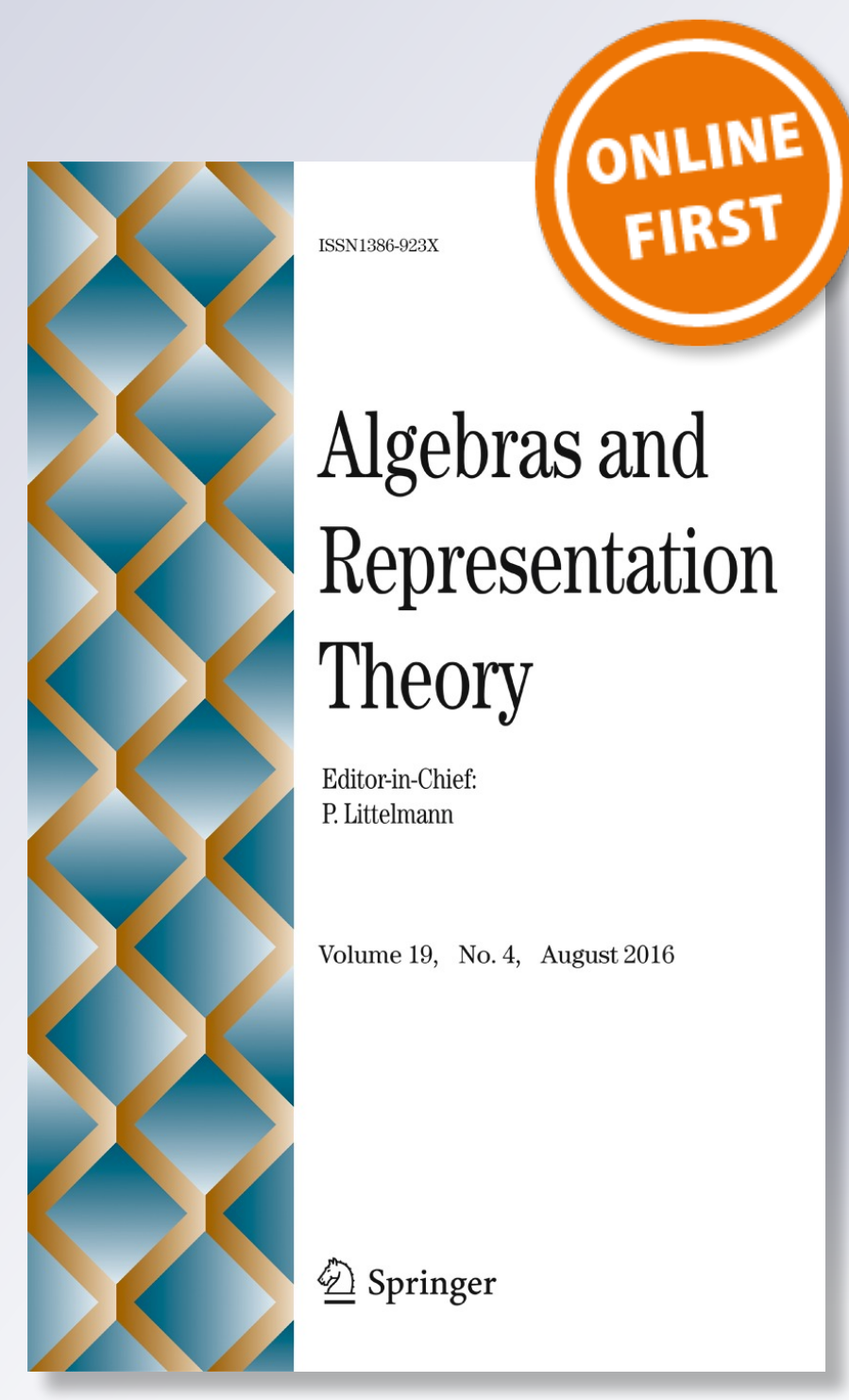

望 Springer 
Your article is protected by copyright and all rights are held exclusively by Springer Science +Business Media Dordrecht. This e-offprint is for personal use only and shall not be selfarchived in electronic repositories. If you wish to self-archive your article, please use the accepted manuscript version for posting on your own website. You may further deposit the accepted manuscript version in any repository, provided it is only made publicly available 12 months after official publication or later and provided acknowledgement is given to the original source of publication and a link is inserted to the published article on Springer's website. The link must be accompanied by the following text: "The final publication is available at link.springer.com". 


\title{
On Sectional Paths in a Category of Complexes of Fixed Size
}

\author{
Claudia Chaio $^{1}$. Isabel Pratti ${ }^{1}$. \\ María José Souto-Salorio ${ }^{2}$
}

Received: 30 December 2015 / Accepted: 20 August 2016

(C) Springer Science+Business Media Dordrecht 2016

\begin{abstract}
We show how to build the Auslander-Reiten quiver of the category $\mathbf{C}_{\mathbf{n}}(\operatorname{proj} \Lambda)$ of complexes of size $n \geq 2$, for any artin algebra $\Lambda$. We also give conditions over the complexes in $\mathbf{C}_{\mathbf{n}}(\operatorname{proj} \Lambda)$ under which the composition of irreducible morphisms in sectional paths vanishes.
\end{abstract}

Keywords Irreducible morphisms · Complexes of fixed size - Auslander-Reiten quiver · Sectional paths

Mathematics Subject Classification (2010) $16 \mathrm{G} 70 \cdot 18 \mathrm{G} 35$

\section{Introduction}

Throughout this paper, unless otherwise stated, all algebras are artin algebras over an artinian (commutative) ring $R$. We furthermore assume that all algebras are basic.

Let $\Lambda$ be an artin algebra and $\bmod \Lambda$ the category of finitely generated right $\Lambda$-modules. We denote by $\operatorname{proj} \Lambda$ the full subcategory of $\bmod \Lambda$ consisting of all projective $\Lambda$-modules.

Presented by Michel Van den Bergh.

María José Souto-Salorio

maria.souto.salorio@udc.es

Claudia Chaio

claudia.chaio@gmail.com

Isabel Pratti

nilprat@mdp.edu.ar

1 Departamento de Matemática, Facultad de Ciencias Exactas y Naturales, Funes 3350,

Universidad Nacional de Mar del Plata, 7600 Mar del Plata, Argentina

2 Departamento de Computación, Facultade de Informática, Universidade da Coruña,

Campus de A Coruña, 15071 A Coruña, España 
The Auslander-Reiten quiver of mod $\Lambda$ (for several algebras) can be constructed by an iterated procedure which was used since 1977. It is named "knitting of components" and the almost split sequences yield to what are called meshes. For any artin algebra, the knitting algorithm constructs inductively the reachable modules, according to their distance to the simple projective modules. Note that the algorithm for constructing reachable modules may stop for two reasons: either we have obtained all the indecomposables or else we encounter an indecomposable direct summand of the radical of an indecomposable projective module such that some other indecomposable direct summand is not reachable or else we encounter an indecomposable direct summand.

The knitting procedure works well in case any indecomposable projective module to be considered has an indecomposable radical (or all the indecomposable direct summands are isomorphic). A criterion for dealing with the case when radical has non-isomorphic indecomposable direct summands was given by the so called s-condition of BautistaLarrion-Salmeron.

Dually, the knitting algorithm can be constructed also backwards starting from the simple injective modules.

In this work we focus our attention on the category $\mathbf{C}_{\mathbf{n}}(\operatorname{proj} \Lambda$ ) (for a positive integer $n \geq 2$ ). This category consists of the complexes $X$, such that $X^{i}=0$ if $i \notin\{1, \ldots, n\}$ and $X^{i} \in \operatorname{proj} \Lambda$ if $X^{i} \neq 0$. In [7], the authors proved that $\mathbf{C}_{\mathbf{n}}(\operatorname{proj} \Lambda)$ is an exact category and has almost split sequences. We recall the main properties in Section 2.

For any artin algebra there is a relationship between the arrows of the quiver and nonzero morphisms between indecomposable projective modules. Moreover, if we deal with a hereditary finite dimensional algebra $H$, over an algebraically closed field, there is an arrow from $i$ to $j$ in the ordinary quiver $Q_{H}$ if and only if there is an irreducible morphism from $P_{j}$ to $P_{i}$ in the Auslander-Reiten quiver of $\bmod H$. In Section 3, we analyze similar results for the category $\mathbf{C}_{\mathbf{n}}(\operatorname{proj} \Lambda)$.

In order to "knit" the Auslander-Reiten quiver of $\mathbf{C}_{\mathbf{n}}(\operatorname{proj} \Lambda)$ following the construction of the corresponding quiver of $\bmod \Lambda$, we first study analogous properties for right almost split morphisms in $\mathbf{C}_{\mathbf{n}}(\operatorname{proj} \Lambda)$. This is the main aim of Section 4. Proposition 4.6 gives a procedure to complete a minimal left almost split morphism $f$ starting at a non-injective to an almost split sequence in $\mathbf{C}_{\mathbf{n}}(\operatorname{proj} \Lambda$ ). In order to do that, we only need to get the complex Coker $f$ from a section $f$. We show the procedure giving an example in Section 5 .

By now, there is a vast literature dealing with translation quivers which arise for artin algebras. For example, one of the basic ideas which R. Bautista introduced in [3], concerns the study of sectional paths and sectional cycles. R. Bautista and S. Smalø, in [5], have shown that an Auslander-Reiten quiver never contains sectional cyclic paths and obtained several important consequences from this fact. In Section 6, we study the behaviour of the compositions of irreducible morphisms in sectional paths. Some of the results can be seen as a "natural" generalization of those in $\bmod \Lambda$ but there are some important differences. We know that in $\bmod \Lambda$, the composition of irreducible morphisms on a sectional path is nonzero, see [5] and [12]. We show that this is not a general fact in $\mathbf{C}_{\mathbf{n}}(\operatorname{proj} \Lambda$ ). More precisely, we can find sectional paths such that their compositions vanish.

\section{Preliminaries}

\section{1}

Let $R$ be a commutative artinian ring and $\mathcal{A}$ a Krull-Schmidt $R$-category, that is, a Homfinite additive $R$-category in which idempotents split. A morphism $f: X \rightarrow Y$ with $X, Y \in$ 
$\mathcal{A}$, is called irreducible provided it does not split and whenever $f=g h$ then either $h$ is a section or $g$ is a retraction.

We say that $f: X \rightarrow Y$ with $X, Y \in \mathcal{A}$ is a minimal left almost split morphism (or a source morphism) if $f$ is not a section, for each morphism $g: X \rightarrow M$ that is not a section $g$ factories through $f$ and if $f=h f$ then $h \in \operatorname{Aut}(Y)$. Dually, a minimal right almost split morphism (or a sink morphism) is defined.

A short exact sequence $X \stackrel{f}{\rightarrow} Y \stackrel{g}{\rightarrow} Z$ in $\mathcal{A}$ is called an almost split sequence if $f$ and $g$ are a source and a sink morphism, respectively. The object $X$ is called the translate of $Z$. We denote by $\tau^{-1} X=Z$ and $\tau Z=X$.

By $\Re_{\mathcal{A}}$ we denote the Jacobson radical of the category $\mathcal{A}$, which is the ideal generated by the non-isomorphisms between indecomposable objects. For $n>1$, we denote by $\Re_{\mathcal{A}}^{n}$ the set of morphisms which are composition of $n$ morphisms lying in $\Re_{\mathcal{A}}$. Finally, $\Re_{\mathcal{A}}^{\infty}=$ $\cap_{n \geq 1} \Re_{\mathcal{A}}^{n}$ is called the infinite radical of $\mathcal{A}$.

If $X$ and $Y$ are indecomposable objects in $\mathcal{A}$ then the set of irreducible morphisms from $X$ to $Y$ can be seen as the quotient $\operatorname{Irr}_{\mathcal{A}}(X, Y)=\Re_{\mathcal{A}}(X, Y) / \mathfrak{R}_{\mathcal{A}}^{2}(X, Y)$. We recall that $\operatorname{Irr}_{\mathcal{A}}(X, Y)$ is a $k_{Y}-k_{X}$-bimodule, where $k_{Z}=\operatorname{End}_{\mathcal{A}}(Z) / \Re_{\mathcal{A}}(Z, Z)$.

By $\Gamma_{\mathcal{A}}$ we denote the Auslander-Reiten quiver of a Krull-Schmidt category $\mathcal{A}$. The valuation of an arrow $X \rightarrow Y$ in $\Gamma_{\mathcal{A}}$ is defined by $\left(\alpha_{X, Y}, \alpha_{X, Y}^{\prime}\right)$, where $\alpha_{X, Y}=\operatorname{dim}_{k_{X}} \operatorname{Irr}_{\mathcal{A}}(X, Y)$ and $\alpha_{X, Y}^{\prime}=\operatorname{dim}_{k_{Y}} \operatorname{Irr}_{\mathcal{A}}(X, Y)$. Furthermore, if $\alpha_{X, Y}=1$ and $\alpha_{X, Y}^{\prime}=1$ then we say that the arrow has trivial valuation and we do not write it. If all arrows in a component $\Gamma \subset \Gamma_{\mathcal{A}}$ have trivial valuation then we say that the component $\Gamma$ has trivial valuation.

By [13, Proposición 2.1] the Auslander-Reiten quiver of $\mathcal{A}$ is a valued translation quiver, that is, if $X$ and $Y$ are indecomposable objects in $\mathcal{A}$ and $Y$ is not injective then, $\operatorname{dim}_{k_{X}} \operatorname{Irr}_{\mathcal{A}}(X, Y)=\operatorname{dim}_{k_{\tau^{-1}(X)}} \operatorname{Irr}_{\mathcal{A}}\left(Y, \tau^{-1}(X)\right)$ and $\operatorname{dim}_{k_{Y}} \operatorname{Irr}_{\mathcal{A}}(X, Y)=$ $\operatorname{dim}_{k_{Y}} \operatorname{Irr}_{\mathcal{A}}\left(Y, \tau^{-1}(X)\right)$.

A dual result holds if $X$ is not projective.

\section{2}

Let $\Lambda$ be an artin algebra and $\bmod \Lambda$ the category of finitely generated right $\Lambda$-modules. We denote by proj $\Lambda$ the full subcategory of $\bmod \Lambda$ consisting of all projective $\Lambda$-modules.

In [7], the authors studied the category $\mathbf{C}_{\mathbf{n}}(\operatorname{proj} \Lambda)$, for $n \in \mathbb{Z}, n \geq 1$, that is, the full subcategory of $\mathbf{C}(\operatorname{proj} \Lambda)$ whose objects are the complexes $X$ with $X^{i}=0$ if $i \notin\{1, \ldots, n\}$. Moreover, given an indecomposable module $P \in \operatorname{proj} \Lambda$, they consider the following complexes:

$$
\begin{aligned}
& J_{i}(P)=\left(J^{s}, d^{s}\right)_{s \in \mathbb{Z}} \text { for } i \in\{1, \cdots, n-1\}, \text { with } J^{s}=0 \text { iff } \neq i, s \neq i+1, J^{i}=J^{i+1} \\
& =P, d^{i}=i d_{P},
\end{aligned}
$$

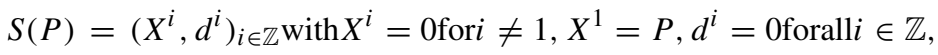

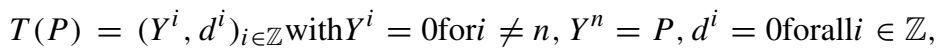

and proved that all the indecomposable projective complexes in $\mathbf{C}_{\mathbf{n}}(\operatorname{proj} \Lambda)$ are of the form $T(P)$ or $J_{i}(P)$ for $i=1, \ldots, n-1$, and all the indecomposable injective complexes in $\mathbf{C}_{\mathbf{n}}(\operatorname{proj} \Lambda)$ are of the form $S(P)$ or $J_{i}(P)$ for $i=1, \ldots, n-1$, see [7, Corollary 3.5]. The authors also proved that $\mathbf{C}_{\mathbf{n}}(\operatorname{proj} \Lambda)$ is an exact category with enough projective and injective objects.

We denote by $X^{1} \stackrel{d^{1}}{\rightarrow} X^{2} \stackrel{d^{2}}{\rightarrow} \cdots \stackrel{d^{n-1}}{\rightarrow} X^{n}$ a complex $X \in \mathbf{C}_{\mathbf{n}}(\operatorname{proj} \Lambda)$. 
For each indecomposable non-projective $X \in \mathbf{C}_{\mathbf{n}}(\operatorname{proj} \Lambda)$, the authors defined the Auslander-Reiten translate of $X$, denoted by $A_{n}(X)$, which they proved that it is also indecomposable in $\mathbf{C}_{\mathbf{n}}(\operatorname{proj} \Lambda)$. By [7, Theorem 8.2], we know that $\mathbf{C}_{\mathbf{n}}(\operatorname{proj} \Lambda)$ has almost split sequences.

\section{3}

We denote by $\overline{\mathbf{C}_{\mathbf{n}}}(\operatorname{proj} \Lambda)$ (respectively, $\underline{\mathbf{C}_{\mathbf{n}}}(\operatorname{proj} \Lambda)$ ) the category whose objects are the same of $\mathbf{C}_{\mathbf{n}}(\operatorname{proj} \Lambda)$ and the morphisms are the morphisms in $\mathbf{C}_{\mathbf{n}}(\operatorname{proj} \Lambda)$ modulo the ones that factor through injective objects (respectively, projective objects).

Consider $X=\left(X^{1} \stackrel{d_{X}}{\rightarrow} X^{2}\right)$ and $Y=\left(Y^{1} \stackrel{d_{Y}}{\rightarrow} Y^{2}\right)$ in $\mathbf{C}_{2}(\operatorname{proj} \Lambda)$. We know that there is a functor $\kappa=C o k: \mathbf{C}_{2}(\operatorname{proj} \Lambda) \rightarrow \bmod \Lambda$ defined as follows; $\kappa(X)=\operatorname{Coker} d_{X}$ if $X$ in $\mathbf{C}_{2}(\operatorname{proj} \Lambda)$, and if $f=\left\{f^{1}, f^{2}\right\}: X \rightarrow Y$ is a morphism in $\mathbf{C}_{2}(\operatorname{proj} \Lambda)$ then $\kappa(f)$ : $\kappa(X) \rightarrow \kappa(Y)$ is the induced morphism. By [4, Proposition 3.3] such a functor induces an equivalence between $\overline{\mathbf{C}}_{2}(\operatorname{proj} \Lambda)$ and $\bmod \Lambda$. For each $M \in \bmod \Lambda$ if we consider $X^{1} \stackrel{d_{X}}{\rightarrow}$ $X^{2} \rightarrow M \rightarrow 0$ a minimal projective presentation of $M$ then the complex $P_{M}=X^{1} \stackrel{d_{X}}{\rightarrow} X^{2}$ is in correspondence with $M$ by the inverse equivalence of $\kappa$.

\section{4}

In [10] and [6] the authors studied the irreducible morphisms in categories of chain complexes taking into account their entries. We recall Corollary 2 and Proposition 3 stated in [10] which shall be important for further purposes.

If $f=\left\{f^{i}\right\}_{i=1}^{n}: X \rightarrow Y$ is an irreducible morphism in $\mathbf{C}_{\mathbf{n}}(\operatorname{proj} \Lambda)$ then, one of these conditions hold:

(sec) For each $i \in\{1, \ldots, n\}$, the morphisms $f^{i}$ are sections in $\operatorname{proj} \Lambda$.

(ret) For each $i \in\{1, \ldots, n\}$, the morphisms $f^{i}$ are retractions in proj $\Lambda$.

(ret-irred-sec) There is an $i \in\{1, \ldots, n\}$ such that $f^{i}$ is irreducible in proj $\Lambda$, the morphisms $f^{j}$ are sections for all $j>i$ and the morphisms $f^{j}$ are retractions for all $j<i$.

\section{5}

Let $P$ be an indecomposable projective $\Lambda$-module, $\cdots \stackrel{d^{-3}}{\rightarrow} R^{-2} \stackrel{d^{-2}}{\rightarrow} R^{-1} \stackrel{d^{-1}}{\rightarrow} P \rightarrow$ $P / \operatorname{rad} P \rightarrow 0$ a minimal projective resolution of $P / \operatorname{rad} P$ in $\bmod \Lambda$ and $0 \rightarrow P / \operatorname{rad} P \rightarrow$ $I^{0} \stackrel{g^{0}}{\rightarrow} I^{1} \stackrel{g^{1}}{\rightarrow} \cdots \stackrel{g^{n-j-1}}{\rightarrow} I^{n-j} \rightarrow \cdots$ a minimal injective co-resolution of $P / \operatorname{rad} P$ in $\bmod \Lambda$, $v$ the Nakayama functor; for $s=0, \cdots, n-j, L^{s}=v^{-1}\left(I^{s}\right)$ and $d_{L}^{s}=v^{-1}\left(g^{s}\right)$. Consider the following morphisms of complexes:

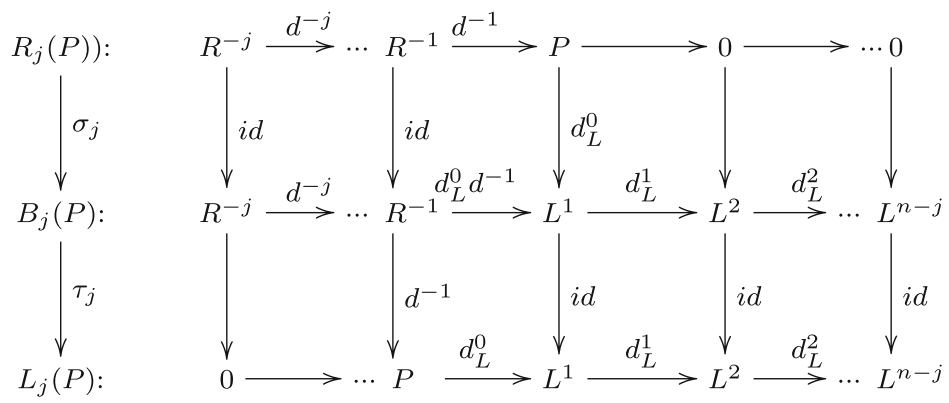


and

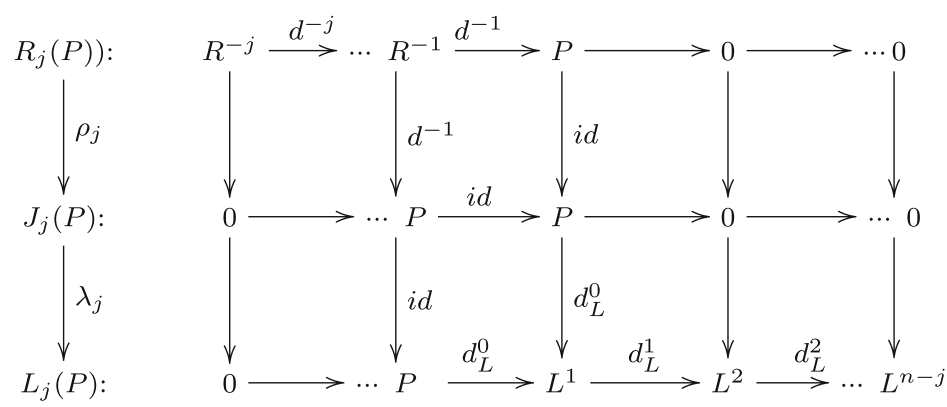

By [7, Proposition 8.5, 8.7, and 8.8] we know that for $j=1, \cdots, n-1$ the morphism $\lambda_{j}: J_{j}(P) \rightarrow L_{j}(P)$ is a minimal left almost split morphism, $\rho_{j}: R_{j}(P) \rightarrow J_{j}(P)$ is a minimal right almost split morphism and $R_{j}(P) \stackrel{\left(\rho_{j}, \sigma_{j}\right)^{t}}{\longrightarrow} J_{j}(P) \oplus B_{j}(P) \stackrel{\left(\lambda_{j}, \tau_{j}\right)}{\longrightarrow} L_{j}(P)$ is an almost split sequence.

\section{Irreducible Morphisms in $C_{n}(\operatorname{proj} \Lambda)$}

We start recalling the fact that if we fix a set of orthogonal primitive idempotents $\left\{e_{1}, \ldots, e_{m}\right\}$ in $\Lambda$, then for each $1 \leq i \leq m$ there is an indecomposable projective $\Lambda$-module $P_{i}=e_{i} \Lambda$ and an indecomposable injective $\Lambda$-module $I_{i}=D\left(\Lambda e_{i}\right)$.

For the convenience of the reader, we state the next well known result from [2, VIII, Proposition 1.15] and [1, VII, Lemma 1.6]), where $Q_{\Lambda}$ denote the ordinary quiver of $\Lambda$.

Proposition 3.1 Let $\Lambda$ be a finite dimensional $k$-algebra over an algebraically closed field $k$. Then, the following conditions hold.

(1) If there is an arrow $i \rightarrow j$ in $Q_{\Lambda}$ then there is a non-zero morphism $P_{j} \rightarrow P_{i}$ in $\bmod \Lambda$.

(2) If there is an irreducible morphism $P_{j} \rightarrow P_{i}$ in $\bmod \Lambda$ then there is an arrow $i \rightarrow j$ in $Q_{\Lambda}$.

Moreover, if $\Lambda$ is an hereditary algebra then the following statements are equivalent:

(3) There is an arrow $i \rightarrow j$ in $Q_{\Lambda}$.

(4) There is an irreducible morphism $P_{j} \rightarrow P_{i}$ in $\bmod \Lambda$.

Our first result shows how the existence of irreducible morphisms in $\mathbf{C}_{\mathbf{n}}(\operatorname{proj} \Lambda)$ is related with the existence of irreducible morphisms in $\bmod \Lambda$.

Proposition 3.2 Let $\Lambda$ be an artin algebra. Consider $P_{i}=e_{i} \Lambda$ an indecomposable projective $\Lambda$-module and $I_{i}=D\left(\Lambda e_{i}\right)$ an indecomposable injective $\Lambda$-module defined as above.

(a) There is an irreducible morphism $T\left(P_{j}\right) \rightarrow T\left(P_{i}\right)$ in $\mathbf{C}_{\mathbf{n}}(\operatorname{proj} \Lambda)$ if and only if there is an irreducible morphism $P_{j} \rightarrow P_{i}$ in $\bmod \Lambda$.

(b) There is an irreducible morphism $S\left(P_{j}\right) \rightarrow S\left(P_{i}\right)$ in $\mathbf{C}_{\mathbf{n}}(\operatorname{proj} \Lambda)$ if and only if there is an irreducible morphism $I_{j} \rightarrow I_{i}$ in $\bmod \Lambda$. 
In case $\Lambda$ is a finite dimensional algebra over an algebraically closed field then the following statements hold.

(c) If there is an arrow $i \rightarrow j$ in $Q_{\Lambda}$ then there is a non-zero morphism $T\left(P_{j}\right) \rightarrow T\left(P_{i}\right)$ in $\mathbf{C}_{\mathbf{n}}(\operatorname{proj} \Lambda)$.

(d) If there is an irreducible morphism $T\left(P_{j}\right) \rightarrow T\left(P_{i}\right)$ in $\mathbf{C}_{\mathbf{n}}(\operatorname{proj} \Lambda)$ then there is an arrow $i \rightarrow j$ in $Q_{\Lambda}$.

Proof $(a)$. Let $f: T\left(P_{j}\right) \rightarrow T\left(P_{i}\right)$ be an irreducible morphism in $\mathbf{C}_{\mathbf{n}}(\operatorname{proj} \Lambda)$. Then, we can assume that $f$ is of the form

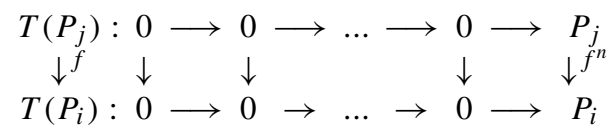

It is clear that $f$ is irreducible in $\mathbf{C}_{\mathbf{n}}(\operatorname{proj} \Lambda)$ if and only if the morphism

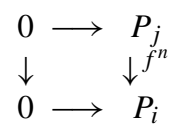

is irreducible in $\mathbf{C}_{2}(\operatorname{proj} \Lambda)$. Moreover, by [4, Lemma 5.1] this fact holds if and only if the morphism $P_{j} \rightarrow P_{i}$ is irreducible in $\bmod \Lambda$.

(b). The morphism $S\left(P_{j}\right) \rightarrow S\left(P_{i}\right)$ is irreducible in $\mathbf{C}_{\mathbf{n}}(\operatorname{proj} \Lambda)$ if and only if the morphism $\left(P_{j} \rightarrow 0\right) \rightarrow\left(P_{i} \rightarrow 0\right)$ is irreducible in $\mathbf{C}_{2}$ (proj $\left.\Lambda\right)$. By [7, Proposition 6.2] we know that $A_{2}\left(P_{j} \rightarrow 0\right)=P_{I_{j}}$, where $P_{I_{j}}$ is the complex defined in (2.3). Hence $A_{2}\left(P_{j} \rightarrow 0\right) \rightarrow A_{2}\left(P_{i} \rightarrow 0\right)$ is irreducible in $\mathbf{C}_{2}(\operatorname{proj} \Lambda)$, that is, $P_{I_{j}} \rightarrow P_{I_{i}}$ is irreducible in $\mathbf{C}_{2}$ (proj $\Lambda$ ). We conclude that $I_{j} \rightarrow I_{i}$ is irreducible in mod $\Lambda$ by using the equivalence between $\bmod \Lambda$ and $\overline{\mathbf{C}}_{2}$ (proj $\Lambda$ ) defined in [4, Proposition 5.9, Lemma 5.3].

(c). By Proposition 3.1, if there is an arrow $i \rightarrow j$ in $Q_{\Lambda}$ then there is a non-zero morphism $f: P_{j} \rightarrow P_{i}$ in $\bmod \Lambda$. Hence, there is a non-zero morphism $\phi: T\left(P_{j}\right) \rightarrow$ $T\left(P_{i}\right)$ in $\mathbf{C}_{\mathbf{n}}(\operatorname{proj} \Lambda)$ where $\phi^{n}=f$ and $\phi^{i}=0$ if $i \neq n$.

$(d)$. If $T\left(P_{j}\right) \rightarrow T\left(P_{i}\right)$ is irreducible in $\mathbf{C}_{\mathbf{n}}(\operatorname{proj} \Lambda)$ then by $(a)$ there is an irreducible morphism $P_{j} \rightarrow P_{i}$ in $\bmod \Lambda$. By Proposition 3.1 we conclude that there is an arrow $i \rightarrow j$ in $Q_{\Lambda}$.

In general, an irreducible morphism $P_{j} \rightarrow P_{i}$ in $\bmod \Lambda$ does not induce an irreducible morphism $S\left(P_{j}\right) \rightarrow S\left(P_{i}\right)$ in $\mathbf{C}_{\mathbf{n}}(\operatorname{proj} \Lambda)$. For example, consider the path algebra of $Q_{\Lambda}$

$$
1 \stackrel{\alpha}{\longrightarrow} 2 \stackrel{\beta}{\longrightarrow} 3
$$

with $\beta \alpha=0$. Assume that an irreducible morphism $P_{3} \rightarrow P_{2}$ in $\bmod \Lambda$ induces an irreducible morphism $g=\left\{g^{1}, 0\right\}:\left(P_{3} \rightarrow 0\right) \longrightarrow\left(P_{2} \rightarrow 0\right)$ in $\mathbf{C}_{2}(\operatorname{proj} \Lambda)$. Hence, $g^{1}$ is neither a retraction nor a section. By [10, Proposition 3, Corollary 2] the morphism $g^{1}$ is irreducible in proj $\Lambda$. Since there is an irreducible morphism $h: P_{2} \rightarrow P_{1}$ in $\operatorname{proj} \Lambda$ and $\operatorname{Hom}_{\Lambda}\left(P_{3}, P_{1}\right)=0$, we infer that $h g^{1}=0$. Therefore, we get the following commutative diagram:

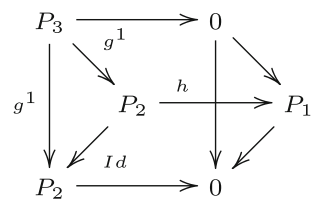


Then, $g=\{i d, 0\}\left\{g^{1}, 0\right\}$. Note that $\{i d, 0\}$ is not a retraction and that $\left\{g^{1}, 0\right\}$ is not a section which contradicts the fact that $g$ is irreducible.

We finish this section with some results for the hereditary case.

Lemma 3.3 Let $H$ be a hereditary artin algebra and $X, Y \in \operatorname{proj} H$. Then, $f: X \rightarrow Y$ is irreducible in proj $H$ if and only if $f: X \rightarrow Y$ is irreducible in mod $H$.

Proof Let $f: X \rightarrow Y$ be an irreducible morphism in proj $H$. Assume that $f=\beta \alpha$ for some $M \in \bmod H$, with $\alpha: X \rightarrow M$ and $\beta: M \rightarrow Y$ in $\bmod H$. Then, $\operatorname{Im} f \subset \operatorname{Im} \beta \in \operatorname{proj} H$. Therefore, $\left.\beta\right|_{M, \operatorname{Im} \beta}: M \rightarrow \operatorname{Im} \beta$ is a retraction. Hence, there is a morphism $\beta^{\prime}: \operatorname{Im} \beta \rightarrow M$ such that $\beta \beta^{\prime}=i d_{\operatorname{Im} \beta}, M=\operatorname{Ker} \beta \oplus \operatorname{Im} \beta^{\prime}$ and $\operatorname{Im} \beta^{\prime} \simeq \operatorname{Im} \beta \in \operatorname{proj} H$. Then, there is a diagram as follows:

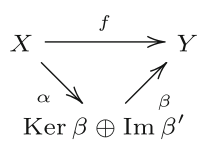

Let $\pi: \operatorname{Ker} \beta \oplus \operatorname{Im} \beta^{\prime} \rightarrow \operatorname{Im} \beta^{\prime}$ be the projection morphism. Consider $\widetilde{\alpha}=\pi \alpha: X \rightarrow$ $\operatorname{Im} \beta^{\prime}$. If $x \in X$ and $\alpha(x)=k+u \in \operatorname{Ker} \beta \oplus \operatorname{Im} \beta^{\prime}$, then $\left.\beta\right|_{\operatorname{Im} \beta^{\prime}} \widetilde{\alpha}(x)=\beta \pi \alpha(x)=\beta(u)=$ $\beta(k+u)=\beta(\alpha(x))=\beta \alpha(x)=f(x)$. Since $f$ is irreducible in proj $H$ then either $\left.\beta\right|_{\operatorname{Im} \beta^{\prime}}$ is a retraction or $\widetilde{\alpha}$ is a section. If $\left.\beta\right|_{\operatorname{Im} \beta^{\prime}}$ is a retraction then $\beta$ is a retraction. Now, if $\widetilde{\alpha}$ is a section then there is a morphism $\tilde{\gamma}: \operatorname{Im} \beta^{\prime} \rightarrow X$ such that $\tilde{\gamma} \widetilde{\alpha}=i d_{X}$. We define the morphism $\gamma: M \rightarrow X$ by $\gamma=\tilde{\gamma} \pi$. Then, $\gamma \alpha=\tilde{\gamma} \pi \alpha=\tilde{\gamma} \widetilde{\alpha}=i d_{X}$. Hence, $\alpha$ is a section and therefore $f: X \rightarrow Y$ is irreducible in $\bmod H$.

The converse follows easily.

Proposition 3.4 Let $H$ be a finite dimensional hereditary algebra over an algebraically closed field. Let $P_{i}$ and $P_{j}$ be the projective $H$-modules corresponding to the vertices $i$ and $j$ in $Q_{H}$, respectively. The following statements are equivalent:

(a) There is an arrow $i \rightarrow j$ in $Q_{H}$.

(b) For every $n \geq 2$, there is an irreducible morphism $T\left(P_{j}\right) \rightarrow T\left(P_{i}\right)$ in $\mathbf{C}_{\mathbf{n}}(\operatorname{proj} H)$.

(c) For every $n \geq 2$, there is an irreducible morphism $S\left(P_{j}\right) \rightarrow S\left(P_{i}\right)$ in $\mathbf{C}_{\mathbf{n}}(\operatorname{proj} H)$.

Proof $(a) \Leftrightarrow(b)$. Since $H$ is hereditary, there is an arrow $i \rightarrow j$ in $Q_{H}$ if and only if there is an irreducible morphism $P_{j} \rightarrow P_{i}$ in $\bmod H$. By Proposition 3.2, $(a), T\left(P_{j}\right) \rightarrow$ $T\left(P_{i}\right)$ is an irreducible morphism in $\mathbf{C}_{\mathbf{n}}(\operatorname{proj} H)$, for any $n \geq 2$. The converse follows by Proposition 3.2, $(d)$.

$(a) \Leftrightarrow(c)$ If there is an arrow $i \rightarrow j$ in $Q_{H}$ then by Proposition 3.1 there is an irreducible morphism $I_{j} \rightarrow I_{i}$ in mod $H$. By Proposition 3.2, (b), we infer the result.

Conversely, if there is an irreducible morphism $f: S\left(P_{j}\right) \rightarrow S\left(P_{i}\right)$ in $\mathbf{C}_{\mathbf{n}}(\operatorname{proj} H)$, by [10, Proposition 3, Corollary 2], we have that $f^{1}: P_{j} \rightarrow P_{i}$ is an irreducible morphism in proj $H$. By Lemma 3.3, $f^{1}: P_{j} \rightarrow P_{i}$ is an irreducible morphism in mod $H$. Then, there is an arrow $i \rightarrow j$ in $Q_{H}$ since $H$ is hereditary.

\section{On Almost Split Sequences}

By [7, Theorem 8.2] we know that there exists a minimal right (left) almost split morphism in $\mathbf{C}_{\mathbf{n}}(\operatorname{proj} \Lambda$ ), ending (starting) at any indecomposable non-projective (non-injective, 
respectively) complex. Moreover, the authors studied the right (left) almost split morphisms in $\mathbf{C}_{\mathbf{n}}$ (proj $\Lambda$ ) ending (starting, respectively) at $J_{i}(P)$, for $P$ an indecomposable projective $\Lambda$-module. We shall complete their study proving the existence of almost split morphisms in $\mathbf{C}_{\mathbf{n}}(\operatorname{proj} \Lambda)$ ending at a projective complex of the form $T(P)$ or starting in an injective complex $S(P)$, for $P$ is an indecomposable projective $\Lambda$-module. These results will allow us to construct the Auslander-Reiten quiver of $\mathbf{C}_{\mathbf{n}}(\operatorname{proj} \Lambda)$.

Remark 4.1 Let $P$ be an indecomposable projective $\Lambda$-module, $\pi: R^{-1} \rightarrow \operatorname{rad} P$ the projective cover of $\operatorname{rad} P$ and $j: \operatorname{rad} P \rightarrow P$ a minimal right almost split morphism in $\bmod \Lambda$. Then, $d^{-1}=j \pi$ is a minimal right almost split morphism in proj $\Lambda$. In fact, consider $\beta: Q \rightarrow P$ a non-split morphism in proj $\Lambda$. Then, there is a morphism $\gamma: Q \rightarrow \operatorname{rad} P$ such that $j \gamma=\beta$. Moreover, since $Q$ is projective and $\pi$ is an epimorphism then there is a morphism $\alpha: Q \rightarrow R^{-1}$ such that $\pi \alpha=\gamma$. Hence, $d^{-1} \gamma=(j \pi) \alpha=j(\pi \alpha)=j \gamma=\beta$, proving that $d^{-1}$ is a right almost split morphism. In order to see that $d^{-1}$ is minimal we consider a morphism $h: R^{-1} \rightarrow R^{-1}$ with $d^{-1} h=d^{-1}$. Then, $j \pi h=j \pi$. Since $j$ is a monomorphism we infer that $\pi h=\pi$. Hence, $\pi h$ is an epimorphism. Moreover, $h$ is an epimorphism and therefore an isomorphism.

Theorem 4.2 Let $P$ be an indecomposable projective $\Lambda$-module. Consider

$$
\cdots \stackrel{d^{-3}}{\rightarrow} R^{-2} \stackrel{d^{-2}}{\rightarrow} R^{-1} \stackrel{d^{-1}}{\rightarrow} P \rightarrow P / \operatorname{rad} P \rightarrow 0
$$

a minimal projective resolution of $P / \operatorname{rad} P$ in $\bmod \Lambda$ and

$$
0 \rightarrow P / \operatorname{rad} P \rightarrow I^{0} \stackrel{g^{0}}{\rightarrow} I^{1} \stackrel{g^{1}}{\rightarrow} \ldots \stackrel{g^{n-j-1}}{\rightarrow} I^{n-j} \rightarrow \cdots
$$

a minimal injective co-resolution of $P / \operatorname{rad} P$ in $\bmod \Lambda$. Let $L^{s}=v^{-1}\left(I^{s}\right)$ and $d_{L}^{s}=$ $v^{-1}\left(g^{s}\right)$ where $v=D \operatorname{Hom}_{\Lambda}(-, \Lambda): \bmod \Lambda \rightarrow \bmod \Lambda$ is the Nakayama functor and $s=0, \cdots, n-j$. The following statements hold.

(a) If $R^{-1} \neq 0$ then the morphism $\rho: R(T(P)) \rightarrow T(P)$ defined as follows

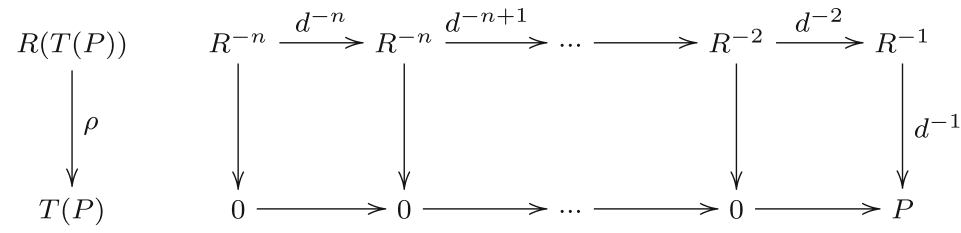

is a minimal right almost split morphism in $\mathbf{C}_{\mathbf{n}}(\operatorname{proj} \Lambda)$.

(b) If $L^{1} \neq 0$, the morphism $\lambda: S(P) \rightarrow L(P)$ given by
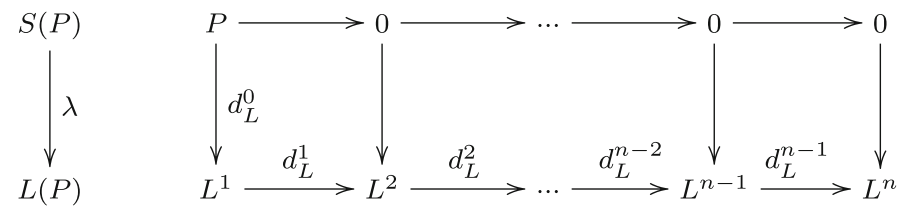

is a minimal left almost split morphism in $\mathbf{C}_{\mathbf{n}}(\operatorname{proj} \Lambda)$. 
Proof $(a)$. By the above remark the morphism $d^{-1}: R^{-1} \rightarrow P$ is irreducible in proj $\Lambda$. Therefore, $\rho$ is neither a section nor a retraction. Consider $g=\left\{g^{i}\right\}_{i=1}^{n}: Q \rightarrow T(P)$ in $\mathbf{C}_{\mathbf{n}}(\operatorname{proj} \Lambda)$ not to be a retraction. We illustrate the situation as follows:

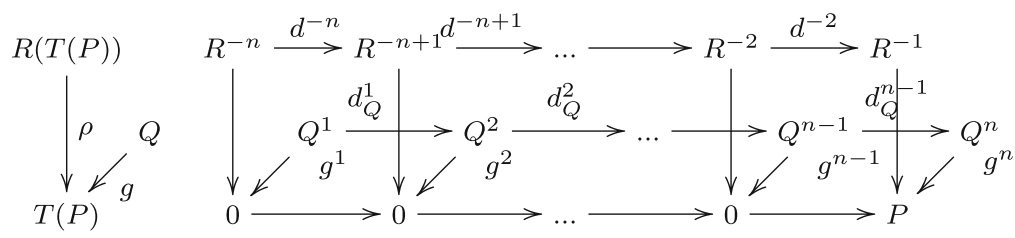

Observe that $g^{i}=0$ if $i \neq n$ and that $g^{n}$ is not a retraction, since otherwise $g$ is a retraction. We shall prove that there is a morphism $\mu: Q \rightarrow R(T(P))$ such that $\rho \mu=g$.

Since $R^{-1} \stackrel{d^{-1}}{\rightarrow} P$ is a right almost split morphism in proj $\Lambda$ there exists a morphism $\mu^{n}: Q^{n} \rightarrow R^{-1}$ such that $d^{-1} \mu^{n}=g^{n}$. Assume that $\mu^{n}, \cdots, \mu^{j}$, are defined for some $1<$ $j \leq n$ and that $d^{n-i+1} \mu^{i}=\mu^{i+1} d_{Q}^{i}$ for $i=j, j+1, \cdots, n$. We write $\alpha=n-j+1$. Next, we prove that we can define $\mu^{j-1}: Q^{j-1} \rightarrow R^{-(\alpha+1)}$ such that $d_{Q}^{-(\alpha+1)} \mu^{j-1}=\mu^{j} d_{Q}^{j-1}$. We recall that $d^{-(\alpha+1)}=i \pi$, where $i: \operatorname{Ker} d^{-\alpha} \rightarrow R^{-\alpha}$ is the inclusion morphism and $\pi: R^{-(\alpha+1)} \rightarrow \operatorname{Ker} d^{-\alpha}$ is the minimal projective cover of Ker $d^{-\alpha}$. Since $d^{-\alpha} \mu^{j} d_{Q}^{j-1}=$ $\mu^{j+1} d_{Q}^{j} d_{Q}^{j-1}=0$ then $\mu^{j} d_{Q}^{j-1}$ factorizes through $\operatorname{Ker} d^{-\alpha}$. That is, there is a morphism $\mu^{\prime}$ : $Q^{j-1} \rightarrow \operatorname{Ker} d^{-\alpha}$ such that $i \mu^{\prime}=\mu^{j} d_{Q}^{j-1}$. Since $Q^{j-1}$ is projective there is a morphism $\mu^{j-1}: Q^{j-1} \rightarrow R^{-(\alpha+1)}$ such that $\pi \mu^{j-1}=\mu^{\prime}$. Then, $i \pi \mu^{j-1}=i \mu^{\prime}$ and we infer that $d^{-(\alpha+1)} \mu^{j-1}=\mu^{j} d_{Q}^{j-1}$. Therefore, we prove the existence of a morphism $\mu: Q \rightarrow$ $R(T(P))$ such that $\rho \mu=g$. Hence, $\rho$ is a right almost split morphism. Furthermore, the fact that $\rho$ is minimal is a consequence that $\cdots \rightarrow R^{-2} \stackrel{d^{-2}}{\rightarrow} R^{-1} \stackrel{d^{-1}}{\rightarrow} P \rightarrow P / \operatorname{rad} P \rightarrow 0$ is a minimal projective resolution of $P / \operatorname{rad} P$.

$(b)$. We get $(b)$ by using the equivalence $v: \mathbf{C}_{\mathbf{n}}(\operatorname{proj} \Lambda) \rightarrow \mathbf{C}_{\mathbf{n}}(\operatorname{inj} \Lambda)$ stated in [7, Sec. 6].

By general Auslander-Reiten theory we know that $\operatorname{Irr}_{\Lambda}(-, S)=0$ if and only if $S$ is a simple projective $\Lambda$-module. Next, we shall prove a similar result for $\mathbf{C}_{\mathbf{n}}(\operatorname{proj} \Lambda)$.

Proposition 4.3 Let $X$ be an indecomposable complex in $\mathbf{C}_{\mathbf{n}}(\operatorname{proj} \Lambda)$. Then, the following conditions hold.

(a) $\operatorname{Irr}_{\mathbf{C}_{\mathbf{n}}(\operatorname{proj} \Lambda)}(-, X)=0$ if and only if $X=T(S)$ for $S$ a simple projective $\Lambda$-module.

(b) $\operatorname{Irr}_{\mathbf{C}_{\mathbf{n}}(\operatorname{proj} \Lambda)}(X,-)=0$ if and only if $X=S(P)$ for $P$ an indecomposable projective $\Lambda$-module such that $v(P)$ is a simple injective, where $v$ is the Nakayama functor.

Proof (a). Let $X$ be an indecomposable complex of the form $J_{i}(P)$ or $T(P)$ where $P$ is not a simple projective $\Lambda$-module. By Theorem $4.2(a)$ and [7, Proposition 8.5] there are irreducible morphisms ending in $X$.

If $X$ is an indecomposable complex, not projective, by [7, Sec. 6 and 8] there is an almost split sequence in $\mathbf{C}_{\mathbf{n}}$ (proj $\Lambda$ ) of the form $A_{n}(X) \rightarrow E \rightarrow X$, and therefore $E \rightarrow X$ is an irreducible morphism ending in $X$. In all these cases, $\operatorname{Irr}_{\mathbf{C}_{\mathbf{n}}(\operatorname{proj} \Lambda)}(-, X) \neq 0$.

Conversely. Assume there is an irreducible morphism $f=\left\{f^{i}\right\}_{i=1}^{n}: X \rightarrow T(S)$ in $\mathbf{C}_{\mathbf{n}}(\operatorname{proj} \Lambda)$ with $S$ a simple projective $\Lambda$-module. Hence, $f^{i}=0$ for $i \neq n$ and $f^{n}$ : 
$X^{n} \rightarrow S$ is a non-zero morphism in $\bmod \Lambda$. Since $S$ is simple projective then $f^{n}$ is a retraction. Hence, $f$ is a retraction contradicting the fact that $f$ is irreducible. Therefore, $\operatorname{Irr}_{\mathbf{C}_{\mathbf{n}}(\operatorname{proj} \Lambda)}(-, T(S))=0$.

(b). Similarly, using Theorem $4.2(b)$, [7, Proposition 8.7] and the equivalence $v$ : $\mathbf{C}_{\mathbf{n}}(\operatorname{proj} \Lambda) \rightarrow \mathbf{C}_{\mathbf{n}}(\operatorname{inj} \Lambda)$ from [7, Sec.6], we get the result.

As an immediate consequence of the above result we get the following corollary.

Corollary 4.4 Let $S$ be a simple projective $\Lambda$-module and $T(S) \rightarrow X$ an irreducible morphism in $\mathbf{C}_{\mathbf{n}}(\operatorname{proj} \Lambda)$ with $X$ indecomposable. Then, $X$ is projective.

The next result in $\mathbf{C}_{\mathbf{n}}(\operatorname{proj} \Lambda)$ is similar to the one given for $\bmod \Lambda$ in $[2, \mathrm{~V}]$.

Proposition 4.5 Let $f: X \rightarrow Y$ be a morphism in $\mathbf{C}_{\mathbf{n}}(\operatorname{proj} \Lambda)$. The following conditions hold.

(a) If $f$ is a right almost split morphism and $Y$ is not projective then $Y$ is indecomposable and $f$ is an epimorphism.

(b) If $f$ is a left almost split morphism and $X$ is not injective then $X$ is indecomposable and $f$ is a monomorphism.

Given $f: X \rightarrow Y$ in $\mathbf{C}_{\mathbf{n}}(\bmod \Lambda)$, we denote by $\operatorname{Ker} f$ the complex with entries $\operatorname{Ker} f^{i}$ and induced differentials. We denote by Coker $f$ the complex with entries Coker $f^{i}$ and induced differentials.

The following result is fundamental to obtain the Auslander-Reiten quiver of $\mathbf{C}_{\mathbf{n}}(\operatorname{proj} \Lambda)$.

Proposition 4.6 Let $\Lambda$ be artin algebra. If $f: X \rightarrow Y$ is a minimal left almost split morphism in $\mathbf{C}_{\mathbf{n}}$ (proj $\Lambda$ ) with $X$ not injective, then $X \rightarrow Y \rightarrow$ Coker $f$ is an almost split sequence in $\mathbf{C}_{\mathbf{n}}(\operatorname{proj} \Lambda)$.

Proof Let $X \stackrel{g}{\rightarrow} E \stackrel{h}{\rightarrow} A_{n}^{-1}(Y)$ be an almost split sequence starting in $X$. Since $f$ and $g$ are minimal left almost split morphisms, then there exists an isomorphism $u: E \rightarrow Y$ such that $u g=f$.

Consider the exact sequence $X \stackrel{f}{\rightarrow} Y \stackrel{t}{\rightarrow}$ Coker $f$ in $\mathbf{C}_{\mathbf{n}}(\bmod \Lambda)$. Since $t u g=0$, there exists a morphism $\alpha: A_{n}^{-1}(Y) \rightarrow \operatorname{Coker} f$ in $\mathbf{C}_{\mathbf{n}}(\bmod \Lambda)$ such that $t u=\alpha h$. We get the following commutative diagram in $\mathbf{C}_{\mathbf{n}}(\bmod \Lambda)$

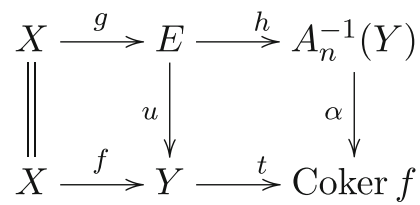

with $\alpha$ an isomorphism. Therefore, Coker $f \simeq A_{n}^{-1}(Y) \in \mathbf{C}_{\mathbf{n}}(\operatorname{proj} \Lambda)$.

The following result is fundamental for our further purposes.

Proposition 4.7 Let $\Lambda$ be an artin algebra and $P$ be an indecomposable projective $\Lambda$ module. The following conditions hold. 
(a) $\operatorname{dim}_{k_{R_{i}(P)}} \operatorname{Irr}_{\mathbf{C}_{\mathbf{n}}(\operatorname{proj} \Lambda)}\left(R_{i}(P), J_{i}(P)\right)=\operatorname{dim}_{J_{i}(P)} \operatorname{Irr}_{\mathbf{C}_{\mathbf{n}}(\operatorname{proj} \Lambda)}\left(R_{i}(P), J_{i}(P)\right)=1$.

(b) $\operatorname{dim}_{k_{i}(P)} \operatorname{Irr}_{\mathbf{C}_{\mathbf{n}}(\operatorname{proj} \Lambda)}\left(J_{i}(P), L_{i}(P)\right)=\operatorname{dim}_{L_{i}(P)} \operatorname{Irr}_{\mathbf{C}_{\mathbf{n}}(\operatorname{proj} \Lambda)}\left(J_{i}(P), L_{i}(P)\right)=1$.

Proof $(a)$. Consider $\rho_{i}: R_{i}(P) \rightarrow J_{i}(P)$ a minimal right almost split morphism. Since $R_{i}(P)$ is indecomposable, then for any irreducible morphism $f: R_{i}(P) \rightarrow$ $J_{i}(P)$ there is an automorphism $\delta: R_{i}(P) \rightarrow R_{i}(P)$ such that $f=\rho_{i} \delta$. Hence, $\operatorname{dim}_{R_{R_{i}(P)}} \operatorname{Irr}_{\mathbf{C}_{\mathbf{n}}(\operatorname{proj} \Lambda)}\left(R_{i}(P), J_{i}(P)\right)=1$.

To prove that $\operatorname{dim}_{J_{i}(P)} \operatorname{Irr}_{\mathbf{C}_{\mathbf{n}}(\operatorname{proj} \Lambda)}\left(R_{i}(P), J_{i}(P)\right)=1$ it is enough to show that $J_{i}(P)$ is not a direct summand of $B_{i}(P)$. Let $\cdots \stackrel{d^{-3}}{\rightarrow} R^{-2} \stackrel{d^{-2}}{\rightarrow} R^{-1} \stackrel{d^{-1}}{\rightarrow} P \rightarrow P / \operatorname{rad} P \rightarrow 0$ be a minimal projective resolution of $P / \operatorname{rad} P$ in $\bmod \Lambda$ and $0 \rightarrow P / \operatorname{rad} P \rightarrow I^{0} \stackrel{g^{0}}{\rightarrow}$ $I^{1} \stackrel{g^{1}}{\rightarrow} \ldots \stackrel{g^{n-j-1}}{\rightarrow} I^{n-j} \rightarrow \cdots$ a minimal injective co-resolution of $P / \operatorname{rad} P$ in $\bmod \Lambda$. For $s=0, \cdots, n-j$ let $L^{s}=v^{-1}\left(I^{s}\right)$ and $d_{L}^{s}=v^{-1}\left(g^{s}\right)$. Then, $B_{i}(P)$ is the complex $R^{-i} \stackrel{d^{-i}}{\longrightarrow} \ldots \longrightarrow R^{-1} \stackrel{d_{L}^{0} d^{-1}}{\longrightarrow} L^{1} \stackrel{d_{L}^{1}}{\longrightarrow} L^{2} \stackrel{d_{L}^{2}}{\longrightarrow} \ldots \longrightarrow L^{n-i}$, see Section 2.5 .

Assume that $J_{i}(P)$ is a direct summand of $B_{i}(P)$. Hence, there exists a section $\gamma$ : $J_{i}(P) \rightarrow B_{i}(P)$. This means that there are retractions $\mu: R^{-1} \rightarrow P$ and $\eta: L^{1} \rightarrow P$ such that $\mu \gamma^{i}=i d_{P}$ and $\eta \gamma^{i+1}=i d_{P}$. Since the following diagram is commutative

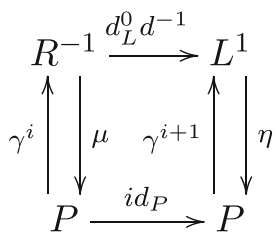

we have that $\mu=\eta d_{L}^{0} d^{-1}$ and $\gamma^{i+1}=d_{L}^{0} d^{-1} \gamma^{i}$. Therefore, $\left(\eta d_{L}^{0}\right)\left(d^{-1} \gamma^{i}\right)=$ $\left(\eta d_{L}^{0} d^{-1}\right) \gamma^{i}=\mu \gamma^{i}=i d_{P}$. As a consequence we get that $\eta d_{L}^{0}: P \rightarrow P$ is a retraction and $d^{-1} \gamma^{i}: P \rightarrow P$ is a section. Moreover, they are isomorphisms. We conclude that $d^{-1}$ is a retraction contradicting that $d^{-1}$ is irreducible in proj $\Lambda$. Then, $\operatorname{dim}_{k_{J_{i}(P)}} \operatorname{Irr}_{\mathbf{C}_{\mathbf{n}}(\operatorname{proj} \Lambda)}\left(R_{i}(P), J_{i}(P)\right)=1$.

$(b)$. Statement $(b)$ follows from the fact that $L_{i}(P)=A_{n}^{-1}\left(R_{i}(P)\right)$ and that $\Gamma_{\mathbf{C}_{\mathbf{n}}(\operatorname{proj} \Lambda)}$ is a valued translation quiver, see [13, Proposition 2.1].

The next result is useful to study the irreducible morphisms ending in a complex $T(P)$.

Lemma 4.8 Let $\Lambda$ be an artin algebra and $P$ an indecomposable non-simple projective $\Lambda$ module. Let $R(T(P))=R^{-n} \rightarrow \cdots \rightarrow R^{-2} \rightarrow R^{-1}$ be the complex in $\mathbf{C}_{\mathbf{n}}(\operatorname{proj} \Lambda)$ defined in Theorem 4.2, (a). If $X^{1} \rightarrow \cdots \rightarrow X^{n-1} \rightarrow X^{n}$ is an indecomposable direct summand of $R(T(P))$ then $X^{n-1} \rightarrow X^{n}$ is an indecomposable direct summand of $R^{-2} \rightarrow R^{-1}$ in $\mathbf{C}_{2}(\operatorname{proj} \Lambda)$.

Proof We know that $\cdots \rightarrow R^{-n} \rightarrow \cdots \rightarrow R^{-2} \rightarrow R^{-1} \rightarrow \operatorname{rad} P \rightarrow 0$ is a minimal projective resolution of $\operatorname{rad} P$. Let $\operatorname{rad} P=\oplus_{i=1}^{s} M_{i}$, with $M_{i}$ indecomposable $\Lambda$-modules for $i=1, \ldots, s$ and $R(T(P))=\bigoplus_{i=1}^{s} P_{M_{i}}$ in $\mathbf{C}_{\mathbf{n}}(\operatorname{proj} \Lambda)$, where $P_{M_{i}}=P_{i}^{1} \rightarrow \cdots \rightarrow$ $P_{i}^{n-1} \rightarrow P_{i}^{n}$ is such that $\cdots \rightarrow P_{i}^{1} \rightarrow \cdots \rightarrow P_{i}^{n-1} \rightarrow P_{i}^{n} \rightarrow M_{i} \rightarrow 0$ is a minimal projective resolution of $M_{i}$, for $i=1, \cdots, s$. Since each $M_{i}$ is indecomposable, then the indecomposable summands of $R(T(P))$ are the complexes $P_{M_{i}}$. Since $P_{i}^{2} \rightarrow P_{i}^{1} \rightarrow M_{i} \rightarrow$ 
0 is a minimal projective presentation of $M_{i}$, then $P_{i}^{2} \rightarrow P_{i}^{1}$ is an indecomposable direct summand of $R^{-2} \rightarrow R^{-1}$ in $\mathbf{C}_{2}(\operatorname{proj} \Lambda)$.

Proposition 4.9 Let $\Lambda$ be an artin algebra. Let $P$ be an indecomposable non-simple projective module and $\operatorname{rad} P=\oplus_{i=1}^{s} M_{i}$, where $M_{i}$ are indecomposable $\Lambda$-modules for $i=1, \ldots, s$. Let $P_{M_{i}}=P_{i}^{1} \rightarrow \cdots \rightarrow P_{i}^{n-1} \rightarrow P_{i}^{n}$ be the complex such that $\cdots \rightarrow P_{i}^{1} \rightarrow \cdots \rightarrow P_{i}^{n-1} \rightarrow P_{i}^{n} \rightarrow M_{i} \rightarrow 0$ is a minimal projective resolution of $M_{i}$, for $i=1, \cdots, s$. Then, the following conditions hold.

(a) $\oplus_{j=1}^{t} P_{M_{i}} \rightarrow T(P)$ is irreducible in $\mathbf{C}_{\mathbf{n}}(\operatorname{proj} \Lambda)$ if and only if $\oplus_{j=1}^{t} M_{i} \rightarrow P$ is irreducible in $\bmod \Lambda$.

(b) $P_{M_{i}} \rightarrow \oplus_{j=1}^{t} T(P)$ is irreducible in $\mathbf{C}_{\mathbf{n}}(\operatorname{proj} \Lambda)$ if and only if $M_{i} \rightarrow \oplus_{j=1}^{t} P$ is irreducible in $\bmod \Lambda$.

Proof (a). The result follows from Theorem 4.2, Lemma 4.8 and the equivalence between $\overline{\mathbf{C}_{2}}(\operatorname{proj} \Lambda)$ and $\bmod \Lambda$ given in (2.3).

(b). If $P_{M_{i}} \rightarrow \oplus_{j=1}^{s} T(P)$ is irreducible in $\mathbf{C}_{\mathbf{n}}(\operatorname{proj} \Lambda)$, by Lemma 4.8 we have that $\left(P_{i}^{n-1} \rightarrow P_{i}^{n}\right) \rightarrow \oplus_{j=1}^{s}(0 \rightarrow P)$ is irreducible in $\mathbf{C}_{2}(\operatorname{proj} \Lambda)$. Hence, $M_{i} \rightarrow \oplus_{j=1}^{s} P$ is irreducible in $\bmod \Lambda$, see (2.3) and [4, Proposition 5.9, Lemma 5.3].

Conversely, if $M_{i} \rightarrow \oplus_{j=1}^{s} P$ is irreducible in $\bmod \Lambda$ then $\left(P_{i}^{n-1} \rightarrow P_{i}^{n}\right) \rightarrow \oplus_{j=1}^{s}(0 \rightarrow$ $P)$ is irreducible in $\mathbf{C}_{\mathbf{2}}(\operatorname{proj} \Lambda)$. Therefore, $P_{M_{i}} \rightarrow \oplus_{j=1}^{s} T(P)$ is irreducible in $\mathbf{C}_{\mathbf{n}}(\operatorname{proj} \Lambda)$.

Dual results hold for irreducible morphisms of the form $S(P) \rightarrow L(P)$, see Theorem 4.2.

Proposition 4.10 Let $\Lambda$ be an artin algebra such that $\Gamma_{\Lambda}$ has trivial valuation. Then, any arrow $X \rightarrow Y$, with $X$ and $Y$ in a pre-projective or a pre-injective component of $\Gamma_{\mathbf{C}_{\mathbf{n}}(\operatorname{proj} \Lambda)}$ has trivial valuation.

Proof We only prove the case where $X$ and $Y$ are pre-projective. If $Y=T(P)$ or $Y=J_{i}(P)$ for some indecomposable projective $\Lambda$-module $P$ then by Proposition 4.7 and Proposition 4.9, we get the result. Otherwise, there is a natural number $m$ such that $A_{n}^{m}(Y)$ is projective. If $A_{n}^{m}(X)$ is defined then as in the above case the arrow $A_{n}^{m}(X) \rightarrow A_{n}^{m}(Y)$ has trivial valuation. Therefore, $X \rightarrow Y$ has also trivial valuation since $\Gamma_{\mathbf{C}_{\mathbf{n}}(\operatorname{proj} \Lambda)}$ is a valued translation quiver. If $A_{n}^{m}(Y)$ is projective then $A_{n}^{j}(X)$ is projective for $j<m$. We get that the arrow $A_{n}^{j+1}(Y) \rightarrow A_{n}^{j}(X)$ has trivial valuation, proving the result.

As an immediate consequence of the above proposition and [2, VII, Proposition 2.3] we get the next result.

Corollary 4.11 Let $\Lambda$ be a finite dimensional algebra over an algebraically closed field of finite representation type. If $\mathbf{C}_{\mathbf{n}}(\operatorname{proj} \Lambda)$ is representation-finite without $A_{n}$-periodic complexes then $\Gamma_{\mathbf{C}_{\mathbf{n}}(\operatorname{proj} \Lambda)}$ has trivial valuation.

Our next result compares the valuation of the arrows in $\Gamma_{\mathbf{C}_{2}(\operatorname{proj} \Lambda)}$ and in $\Gamma_{\Lambda}$.

Proposition 4.12 Let $\Lambda$ be an artin algebra. The following conditions hold. 
(i) If an arrow $X \rightarrow Y$ in $\Gamma_{\Lambda}$ has valuation $(a, b)$ then there is an arrow $P_{X} \rightarrow P_{Y}$ with valuation $(a, b)$ in $\Gamma_{\mathbf{C}_{2}}$ (proj $\Lambda$ ), where $P_{X}$ is defined in (2.3).

(ii) If $\left(X^{1} \stackrel{d_{X}}{\rightarrow} X^{2}\right) \rightarrow\left(Y^{1} \stackrel{d_{Y}}{\rightarrow} Y^{2}\right)$ has valuation $(a, b)$ in $\Gamma_{\mathbf{C}_{2}(\operatorname{proj} \Lambda)},\left(X^{1} \stackrel{d_{X}}{\rightarrow} X^{2}\right)$ and $\left(Y^{1} \stackrel{d_{Y}}{\rightarrow} Y^{2}\right)$ do not have injective direct summands then $X \rightarrow Y$ in $\Gamma_{\Lambda}$ has valuation $(a, b)$, where $X=$ Coker $d_{X}$ and $Y=\operatorname{Coker} d_{Y}$.

(iii) $\Gamma_{\Lambda}$ has trivial valuation if and only if $\Gamma_{\mathbf{C}_{2}(\operatorname{proj} \Lambda)}$ has trivial valuation.

Proof By [4, Lemma 5.1] we get Statements (i) and (ii). Statement (iii) follows from Proposition 4.7, Proposition 4.9, (i) and (ii).

\section{The Auslander-Reiten Quiver of $C_{n}(\operatorname{proj} \Lambda)$}

In general, the Auslander-Reiten quiver of $\mathbf{C}_{\mathbf{n}}(\operatorname{proj} \Lambda)$ is not easy to build, since we must know all the almost split sequences in the category. The above results allow us to build such a quiver by using the same "knitting technique" that we use to build $\Gamma_{\Lambda}$. As in the module category, the mentioned technique is efficient for finite acyclic components of the Auslander-Reiten quiver.

Consider the function of complexes, defined by W. Weeler in [14], that sends a complex $X=\left(X^{1} \rightarrow \cdots \rightarrow X^{n}\right) \in \mathbf{C}_{\mathbf{n}}(\operatorname{proj} \Lambda)$ to the length of a composition series of $\oplus_{i=1}^{n} X^{i}$. Observe that all complexes in $\mathbf{C}_{\mathbf{n}}(\operatorname{proj} \Lambda)$ have bounded length. Then, for a category $\mathbf{C}_{\mathbf{n}}(\operatorname{proj} \Lambda)$ we can prove an analogous of Auslander's theorem that states that if there is a finite connected component $\Gamma$ in the Auslander-Reiten quiver of $\bmod \Lambda$ then $\Gamma$ is the whole Auslander-Reiten quiver.

Example 5.1 Consider the path algebra of the quiver $Q_{\Lambda}$

$$
1 \stackrel{\alpha}{\longrightarrow} 2 \stackrel{\beta}{\longrightarrow} 3
$$

with $\beta \alpha=0$. The Auslander-Reiten quiver of $\bmod \Lambda$ is:

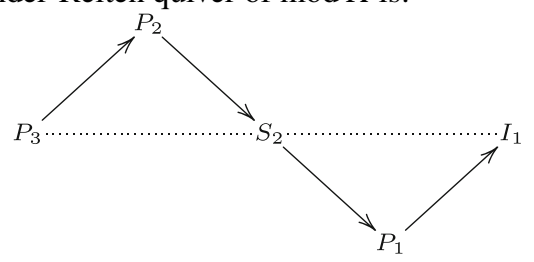

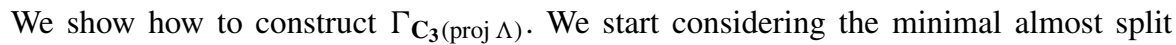
morphisms from $R_{j}\left(P_{i}\right)$ to $J_{j}\left(P_{i}\right)$ for $i=1,2,3$ and $j=1,2$ and from $R\left(T\left(P_{i}\right)\right)$ to $T\left(P_{i}\right)$ for $i=1,2,3$. We compute such complexes in the next table.

$$
\begin{array}{c|c|c|c}
R_{2}\left(P_{i}\right) & P_{3} \rightarrow P_{2} \rightarrow P_{1} & 0 \rightarrow 0 \rightarrow P_{3} & 0 \rightarrow P_{3} \rightarrow P_{2} \\
\hline J_{2}\left(P_{i}\right) & 0 \rightarrow P_{1} \rightarrow P_{1} & 0 \rightarrow P_{3} \rightarrow P_{3} & 0 \rightarrow P_{2} \rightarrow P_{2} \\
R_{1}\left(P_{i}\right) & P_{2} \rightarrow P_{1} \rightarrow 0 & P_{3} \rightarrow P_{2} \rightarrow 0 & 0 \rightarrow P_{3} \rightarrow 0 \\
\hline J_{1}\left(P_{i}\right) & P_{1} \rightarrow P_{1} \rightarrow 0 & P_{2} \rightarrow P_{2} \rightarrow 0 & P_{3} \rightarrow P_{3} \rightarrow 0 \\
R\left(T\left(P_{i}\right)\right) & 0 \rightarrow 0 \rightarrow P_{3} & 0 \rightarrow P_{3} \rightarrow P_{2} \\
\hline T\left(P_{i}\right) & 0 \rightarrow 0 \rightarrow P_{2} & 0 \rightarrow 0 \rightarrow P_{1}
\end{array}
$$

By Proposition 4.7, the valuation of the arrows from $R_{j}\left(P_{i}\right)$ to $J_{j}\left(P_{i}\right)$ for $i=1,2,3$ and $j=1,2$ is trivial. Moreover, by Proposition 4.9 since $\Gamma_{\Lambda}$ has trivial valuation then the 
arrows from the indecomposable direct summands of $R\left(T\left(P_{i}\right)\right)$ to $T\left(P_{i}\right)$ have also trivial valuation for $i=1,2,3$.

To start knitting the Auslander-Reiten quiver first we consider the complex $T\left(P_{3}\right):(0 \rightarrow$ $0 \rightarrow P_{3}$ ). By Proposition 4.3 there are no morphisms ending in this complex, since $P_{3}$ is a simple projective. Then, we get the following subquiver:

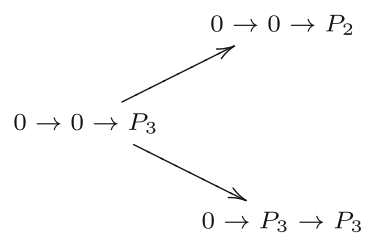

By Corollary 4.4 if there is a morphism $T\left(P_{3}\right) \rightarrow X$ then $X$ is projective. Moreover, the morphism in (1) is a minimal left almost split morphism. By Proposition 4.6, we get an almost split sequence as follows:

$$
\left(0 \rightarrow 0 \rightarrow P_{2}\right) \longrightarrow\left(0 \rightarrow 0 \rightarrow P_{3}\right) \oplus\left(0 \rightarrow P_{3} \rightarrow P_{2}\right) \longrightarrow\left(0 \rightarrow P_{3} \rightarrow P_{3}\right) .
$$

Now, we consider the irreducible morphisms from $\left(0 \rightarrow P_{3} \rightarrow P_{2}\right)$ to the projective complexes $\left(0 \rightarrow 0 \rightarrow P_{1}\right)$ and $\left(0 \rightarrow P_{2} \rightarrow P_{2}\right)$ and since there exists the translate of $\left(0 \rightarrow 0 \rightarrow P_{2}\right)$ we get the subquiver:

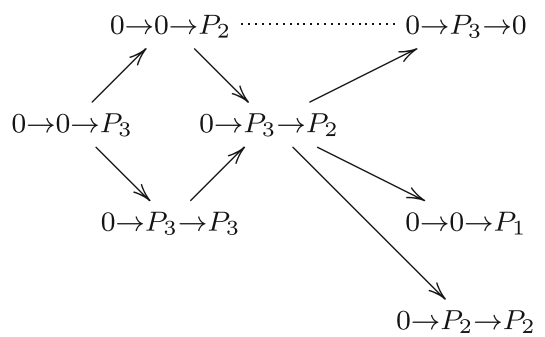

Assume there is another arrow from $\left(0 \rightarrow P_{3} \rightarrow P_{2}\right)$ to $X$. Since $X \neq\left(0 \rightarrow 0 \rightarrow P_{1}\right)$, $X \neq\left(0 \rightarrow P_{2} \rightarrow P_{2}\right)$ and $X \neq\left(0 \rightarrow P_{3} \rightarrow 0\right)$, because such arrows have trivial valuation then $X$ is not projective. Therefore, there is an arrow $A(X) \rightarrow\left(0 \rightarrow P_{3} \rightarrow P_{2}\right)$ contradicting that (2) is an almost split sequence and proving that $\left(0 \rightarrow P_{3} \rightarrow P_{2}\right) \longrightarrow$ $\left(0 \rightarrow P_{3} \rightarrow 0\right) \oplus\left(0 \rightarrow 0 \rightarrow P_{1}\right) \oplus\left(0 \rightarrow P_{2} \rightarrow P_{2}\right)$ is a minimal left almost split morphism. Iterating the above construction in each step we get the Auslander-Reiten quiver of $\mathbf{C}_{\mathbf{3}}(\operatorname{proj} \Lambda)$.

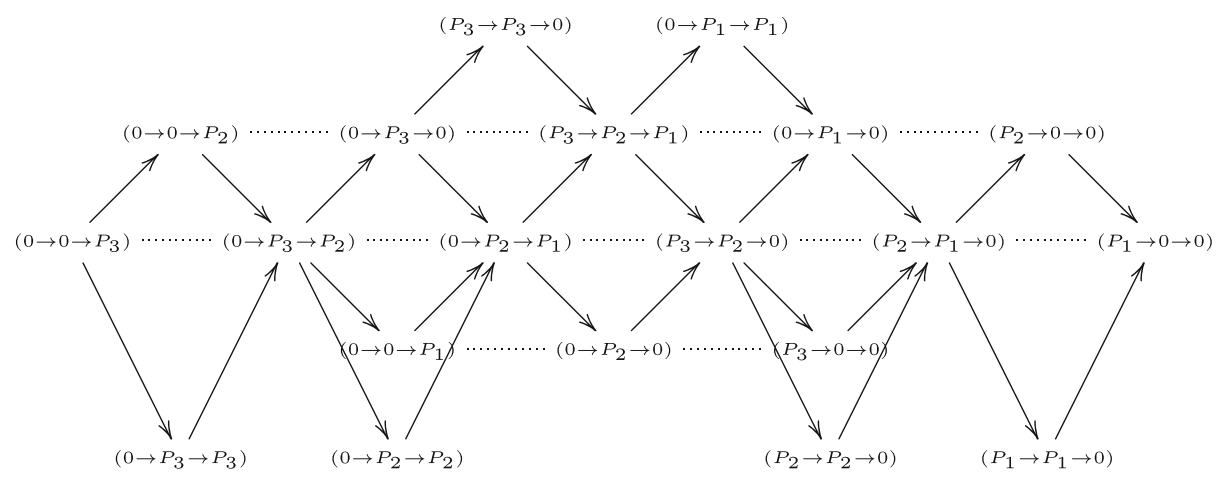


In [7], the authors show that the study of the morphisms in $\mathbf{D}^{b}(\bmod \Lambda)$ can be replaced by the study of morphisms between complexes in $\mathbf{C}_{\mathbf{n}}$ ( $\operatorname{proj} \Lambda$ ) see [11] for a detail account on the derived category. More precisely, they proved that, if $f$ is irreducible in $\mathbf{D}^{b}(\bmod \Lambda)$ then there exists an $n$ such that $f$ is irreducible in $\mathbf{C}_{\mathbf{n}}(\operatorname{proj} \Lambda)$. Similarly, an Auslander-Reiten triangle of $\mathbf{D}^{b}(\bmod \Lambda)$ can be seem as an almost split sequence of $\mathbf{C}_{\mathbf{n}}(\operatorname{proj} \Lambda)$. Next, we illustrate this fact with an example. First, we recall the following result from [6, Corollary 4.5].

Corollary 5.2 [6] Let $X$ and $Y$ be complexes without injective direct summands, $Y$ indecomposable with $Y^{1}=0=Y^{n}$ and $f: X \rightarrow Y$ irreducible in $\mathbf{C}_{\mathbf{n}}(\operatorname{proj} \Lambda)$. Then, $f$ is irreducible in $\boldsymbol{K}^{-, b}\left(\operatorname{proj} \Lambda\right.$ ) if and only if $d_{X}^{1}$ is a monomorphism.

Notation We denote by $P_{1}^{n_{1}} P_{2}^{n_{2}} \cdots P_{h}^{n_{h}}$ the direct sum $\bigoplus_{i=1}^{h} P_{i}^{n_{i}}$ and by $P_{1}^{n_{1}} \cdots P_{h}^{n_{h}}-$ $R_{1}^{s_{1}} \cdots R_{t}^{s_{t}}-Q_{1}^{m_{1}} \cdots Q_{r}^{m_{r}}$ the complex $\bigoplus_{i=1}^{h} P_{i}^{n_{i}} \rightarrow \bigoplus_{i=1}^{t} R_{i}^{s_{i}} \rightarrow \bigoplus_{i=1}^{r} Q_{i}^{m_{i}}$ of $\mathbf{C}_{3}(\operatorname{proj} \Lambda)$.

Example 5.3 Consider $\Lambda$ the path algebra given by the quiver

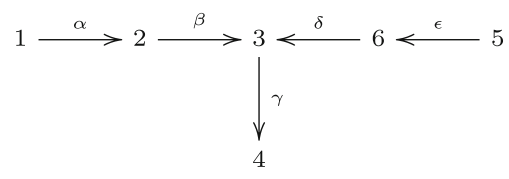

with $\gamma \beta \alpha=0$ and $\gamma \delta \epsilon=0$.

The Auslander Reiten quiver of $\mathbf{C}_{\mathbf{5}}(\operatorname{proj} \Lambda$ ) has the following subquiver (we denote by $j$ the complex $P_{j}$ ):

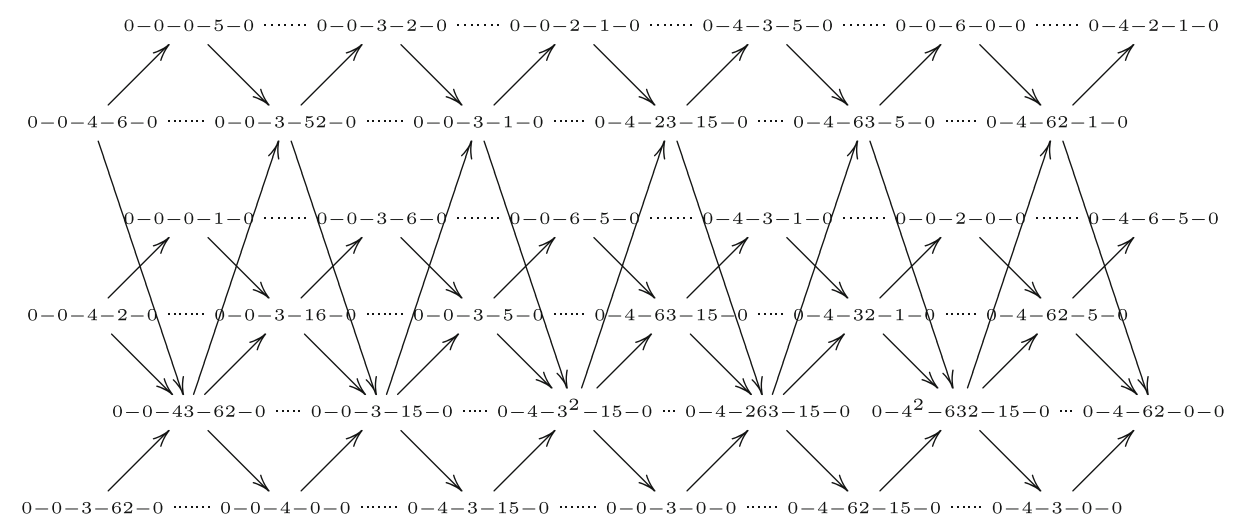

By the above corollary all the irreducible morphisms of such a subquiver are irreducible in $\mathbf{K}^{-, b}(\operatorname{proj} \Lambda)$ and therefore in $\mathbf{D}^{b}(\bmod \Lambda)$.

\section{Sectional Paths}

Some of the results in this section can be seen as a "natural" generalization of those in $\bmod \Lambda$, but there are some important differences. We know that the composition of irreducible morphisms on a sectional path in $\bmod \Lambda$ is non-zero. This result was first proved by R. Bautista and S. Smal $\varnothing$ in 1983, see [5]. At the same time, H. Igusa and G. Todorov 
also studied sectional paths in $\bmod \Lambda$ and proved that the composition of $m$ irreducible morphisms on a sectional path does not belong to $\Re^{m+1}(\bmod \Lambda)$ and as a consequence does not vanish. In this section we prove that, in general, such a property is not true in $\mathbf{C}_{\mathbf{n}}(\operatorname{proj} \Lambda)$.

We start recalling the notion of sectional path.

Definition 6.1 A path of irreducible morphisms between indecomposable complexes in $\mathbf{C}_{\mathbf{n}}(\operatorname{proj} \Lambda) X_{0} \longrightarrow X_{1} \longrightarrow \cdots \longrightarrow X_{m}$ is sectional if $A_{n}^{-1} X_{i} \not X_{i+2}$ for every $i=$ $0, \ldots, m-2$.

The following example shows that there are irreducible morphisms in $\mathbf{C}_{\mathbf{n}}(\operatorname{proj} \Lambda)$ which belong to a sectional path with zero composition.

Example 6.2 Let $\Lambda$ be the path algebra of the quiver $Q_{\Lambda}: 1 \stackrel{\alpha}{\rightarrow} 2 \stackrel{\beta}{\rightarrow} 3 \stackrel{\gamma}{\rightarrow} 4$ with $\beta \alpha=\gamma \beta=0$. The Auslander-Reiten quiver of $\mathbf{C}_{\mathbf{3}}(\operatorname{proj} \Lambda)$ is the following

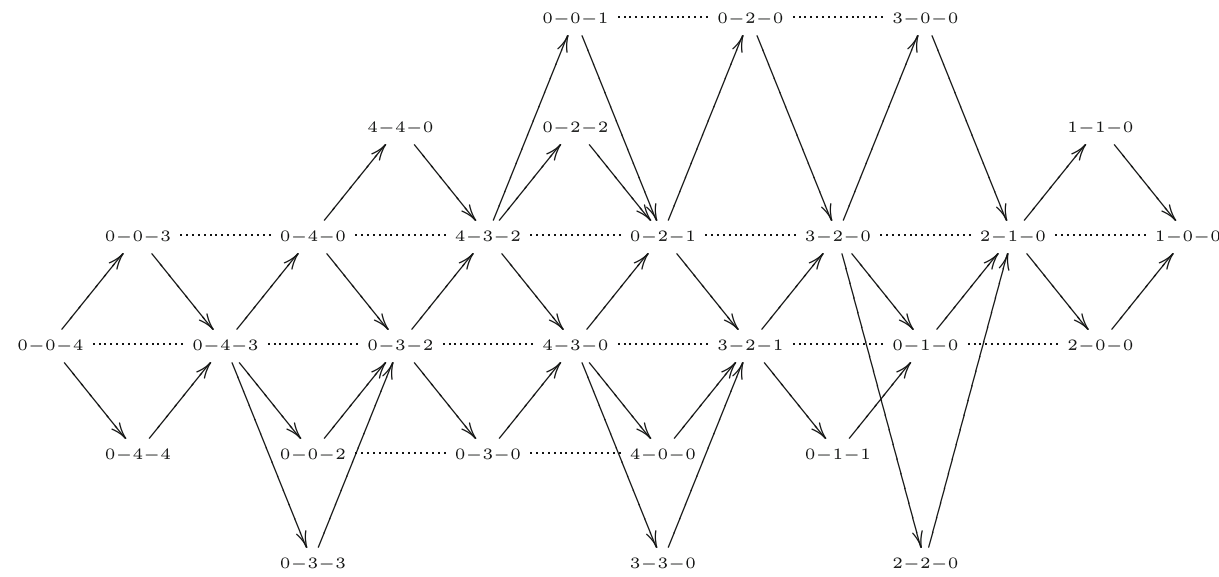

and the paths

$$
\begin{aligned}
J_{2}\left(P_{4}\right) & =\left(0-P_{4}-P_{4}\right) \longrightarrow\left(0-P_{4}-P_{3}\right) \longrightarrow T\left(P_{2}\right)=\left(0-0-P_{2}\right), \\
J_{1}\left(P_{4}\right) & =\left(P_{4}-P_{4}-0\right) \longrightarrow\left(P_{4}-P_{3}-P_{2}\right) \longrightarrow J_{2}\left(P_{2}\right)=\left(0-P_{2}-P_{2}\right), \\
S\left(P_{3}\right) & =\left(P_{3}-0-0\right) \longrightarrow\left(P_{2}-P_{1}-0\right) \longrightarrow J_{1}\left(P_{1}\right)=\left(P_{1}-P_{1}-0\right),
\end{aligned}
$$

are zero sectional paths.

In [13], S. Liu studied the behaviour of sectional paths in left or right Auslander-Reiten categories, $\mathcal{A}$. More precisely, he proved that any sectional path $X_{0} \stackrel{f_{1}}{\rightarrow} X_{1} \stackrel{f_{2}}{\rightarrow} \cdots \stackrel{f_{m}}{\rightarrow} X_{m}$ in these categories verify that $f_{m} \cdots f_{1} \notin \Re_{\mathcal{A}}^{m+1}\left(X_{0}, X_{m}\right)$, see [13, Lemma 2.7].

Now, we consider a hereditary artin algebra $H$. In this case we shall prove that $\mathbf{C}_{\mathbf{n}}(\operatorname{proj} H)$ is a left Auslander-Reiten category. As an immediate consequence we get that the composition of irreducible morphisms in a sectional path is always non-zero.

We recall the following definitions from [13]. 
Definition 6.3 An object $X$ in $\mathbf{C}_{\mathbf{n}}(\operatorname{proj} \Lambda)$ is called pseudo-projective if there exists a sink monomorphism $M \rightarrow X$, and dually, it is called pseudo-injective if there exists a source epimorphism $X \rightarrow N$.

Definition 6.4 A Krull-Schmidt category $\mathcal{A}$ is called a left Auslander Reiten category if each indecomposable object in $\mathcal{A}$ is either pseudo-projective or the end-term of an almost split sequence. $\mathcal{A}$ is called a right Auslander-Reiten category if each indecomposable object in $\mathcal{A}$ is either pseudo-injective or the starting term of an almost split sequence. $\mathcal{A}$ is called an Auslander-Reiten category if it is a left and a right Auslander-Reiten category.

Proposition 6.5 Let $\Lambda$ be an artin algebra. The category $\mathbf{C}_{\mathbf{n}}(\operatorname{proj} \Lambda)$ is a left AuslanderReiten category if and only if $\Lambda$ is hereditary.

Proof Assume that $\Lambda$ is not hereditary. Then, there exists an indecomposable projective $\Lambda$ module $P$ such that the projective dimension of $P / \operatorname{rad} P$ is greater than one. Therefore, we infer that $\mathbf{C}_{\mathbf{n}}$ (proj $\Lambda$ ) is not a left Auslander-Reiten category since the irreducible morphism $\rho: R(T(P)) \rightarrow T(P)$ is not a sink monomorphism and clearly $T(P)$ is not the ending of an almost split sequence.

Now, let $\Lambda$ be a hereditary algebra. Consider $X$ an indecomposable complex in $\mathbf{C}_{\mathbf{n}}(\operatorname{proj} \Lambda)$. If $X$ is not projective then $X$ is an end-term of an almost split sequence. If $X=T(P)$ (or $X=J_{i}(P)$ ) then $\rho: R(T(P)) \rightarrow T(P)$ (or $\rho_{i}: R_{i}(P) \rightarrow J_{i}(P)$ ) is a sink morphism ending in $X$. Since $\Lambda$ is hereditary then the above sink morphisms are monomorphisms. In fact, for every $P \in \operatorname{proj} H$ we have that the projective dimension of $P / \operatorname{rad} P$ is less than or equal to one. We conclude that, $d^{-1}: R^{-1} \rightarrow P$ is a monomorphism and $R^{j}=0$ for every $j<1$, proving the result.

Corollary 6.6 Let $H$ be a hereditary algebra and $X_{0} \rightarrow X_{1} \rightarrow \cdots \rightarrow X_{m}$ be a sectional path in $\mathbf{C}_{\mathbf{n}}(\operatorname{proj} H)$. If $f_{i}: X_{i-1} \rightarrow X_{i}$ are irreducible morphisms for $i=1, \cdots, m$ then $f_{m} \cdots f_{1} \notin \Re_{\mathbf{C}_{\mathbf{n}}(\operatorname{proj} H)}^{m+1}\left(X_{0}, X_{m}\right)$ In particular, $f_{m} \cdots f_{1} \neq 0$.

Next, we show an example of a category $\mathbf{C}_{\mathbf{n}}(\operatorname{proj} H)$, with $H$ a hereditary algebra, which is not a right Auslander-Reiten category.

Example 6.7 Consider $H$ the path algebra given by the quiver

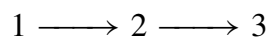

The Auslander-Reiten quiver of $\mathbf{C}_{\mathbf{2}}(\operatorname{proj} H)$ is the following:

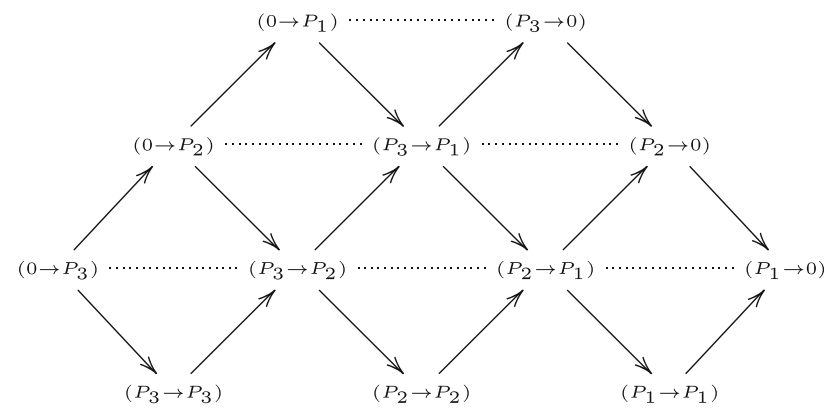


We observe that there is not a source epimorphism starting in the injective $\left(P_{3} \rightarrow 0\right)$ since the irreducible morphism $\left(P_{3} \rightarrow 0\right) \rightarrow\left(P_{2} \rightarrow 0\right)$ is a monomorphism and, furthermore there is not an almost split sequence starting in such a complex. Therefore, $\mathbf{C}_{\mathbf{2}}(\operatorname{proj} H)$ is not a right Auslander-Reiten category.

The next result is a consequence of Proposition 4.7 and the shape of an almost split sequence in $\mathbf{C}_{\mathbf{n}}(\operatorname{proj} \Lambda)$ with an indecomposable projective-injective direct summand of its middle term.

Lemma 6.8 If $X_{0} \rightarrow X_{1} \rightarrow \cdots \rightarrow X_{m}$ is a sectional path with $X_{i}=J_{t}(P)$ for some $i \in\{0, \cdots, m\}$ and where $P$ is an indecomposable projective module then $i=0$ or $i=m$.

Lemma 6.9 Let $\Lambda$ be an artin algebra and $X_{0} \rightarrow X_{1} \rightarrow \cdots \rightarrow X_{m}$ be a non-zero sectional path in $\mathbf{C}_{\mathbf{n}}(\operatorname{proj} \Lambda)$, with $m>1$. Then, one of the following statements hold.

(a) The complexes $X_{1}, X_{2}, \cdots, X_{m}$ are not projective.

(b) The complexes $X_{0}, X_{1}, \cdots, X_{m-1}$ are not injective.

(c) $X_{0}=J_{t}(P)$ and $X_{m}=J_{s}(Q)$ with $P, Q$ indecomposable projective $\Lambda$-modules, $1 \leq t, s \leq m-1$ with $s=t$ or $s+1=t$. Moreover, $X_{1}, \cdots, X_{m-1}$ are neither projective nor injective.

Proof Let $X_{0} \rightarrow X_{1} \rightarrow \cdots \rightarrow X_{m}$ be a non-zero sectional path. If there are no projectives (no injectives, respectively) in such a path then condition $(a)((b)$, respectively) holds. Assume $i \in\{0, \cdots, m\}$ is the least integer such that $X_{i}$ is projective or injective.

Case 1: If $X_{i}=S(Q)$ then $X_{j}$ are not projective for $j>i$, otherwise the path is zero.

Case 2: If $X_{i}=J_{t}(Q)$, by Lemma 6.8 we have that $i=0$ or $i=m$. If $i=0$ then for $j>0$ the complexes $X_{j}$ are not projective of the form $T(P)$, since otherwise the path is zero. We have the following two situations: the path is of the form $J_{t}(Q)=X_{0} \rightarrow \cdots \rightarrow$ $X_{m}=J_{s}(P)$, where $X_{j}$ are neither projective nor injective for $j=1, \ldots, m-1$, and $s=t$ or $s+1=t$ or of the form $J_{t}(Q)=X_{0} \rightarrow \cdots \rightarrow X_{m}$ with $X_{1}, \cdots, X_{m}$ not projective.

If $i=m$ then $X_{0}, \cdots, X_{m-1}$ are not injective.

Case 3: If $X_{i}=T(Q)$ then for $j>i$ the complexes $X_{j}$ are different from $S(P)$, since the path is non-zero. If there is a complex of the form $J_{S}(P)$ then by Lemma 6.8 we have that $s=m$. In this case, $X_{0}, \cdots, X_{m-1}$ are not injective.

Our next aim is to show conditions under which the composition of irreducible morphisms on a sectional path in $\mathbf{C}_{\mathbf{n}}\left(\operatorname{proj} \Lambda\right.$ ) does not belong to $\Re_{\mathbf{C}_{\mathbf{n}}(\operatorname{proj} \Lambda)}^{m+1}$ whenever $\Lambda$ is an artin algebra. We start adapting [12, Lemma 13.2] to $\mathbf{C}_{\mathbf{n}}(\operatorname{proj} \Lambda)$. The proof follows by induction on the length of the sectional path. We state the result below. A dual result holds.

Proposition 6.10 Let $X_{0} \rightarrow X_{1} \rightarrow \cdots \rightarrow X_{m}$ be a sectional path in $\mathbf{C}_{\mathbf{n}}(\operatorname{proj} \Lambda)$ with $X_{2}, \cdots, X_{m}$ non-projective complexes. If $f_{i}: X_{i-1} \rightarrow X_{i}$ are irreducible morphisms for $i=1, \cdots, m$ then there do not exist morphisms $g: X_{0} \rightarrow Z$ and $f_{m}^{\prime}: Z \rightarrow X_{m}$ such that $\left(f_{m} f_{m}^{\prime}\right): Z \oplus X_{m-1} \rightarrow X_{m}$ is a right almost split morphism and $f_{m}^{\prime} g+f_{m} \cdots f_{1} \in$ $\Re_{\mathbf{C}_{\mathbf{n}}(\operatorname{proj} \Lambda)}^{m+1}\left(X_{0}, X_{m}\right)$. 
In [8, Corollary 3.3] the authors studied when the composition of irreducible morphisms between indecomposable modules in a generalized standard component with length belongs to the $n+1$ power of the radical of their module category. Such a result can be adapted to similar components in $\Gamma_{\mathbf{C}_{\mathbf{n}}(\operatorname{proj} \Lambda)}$. More precisely, if $\Gamma \subset \Gamma_{\mathbf{C}_{\mathbf{n}}(\operatorname{proj} \Lambda)}$ is a generalized standard component with length then the composition $f$ of $m$ irreducible morphisms between indecomposable complexes in $\Gamma$ belongs to $\Re_{\mathbf{C}_{\mathbf{n}}(\operatorname{proj} \Lambda)}^{m+1}$ if and only if $f=0$. As an immediate consequence we get the following result.

Proposition 6.11 Let $\Lambda$ be an artin algebra and $\Gamma \subset \Gamma_{\mathbf{C}_{\mathbf{n}}(\operatorname{proj} \Lambda)}$ a generalized standard component with length. Let $X_{0} \rightarrow X_{1} \rightarrow \cdots \rightarrow X_{m}$ be a sectional path with $X_{i} \in \Gamma$ for $i=0, \ldots, m$ with $m \geq 3$. For each irreducible morphism $f_{i}: X_{i-1} \rightarrow X_{i}$ with $i \in\{1, \cdots, m\}$ we have that $f_{m} \cdots f_{1} \in \mathfrak{R}_{\mathbf{C}_{\mathbf{n}}(\operatorname{proj} \Lambda)}^{m+1}\left(X_{0}, X_{m}\right)$ if and only if $f_{m} \cdots f_{1}=0$.

Theorem 6.12 Let $\Lambda$ be an artin algebra. Consider a sectional path $X_{0} \rightarrow X_{1} \rightarrow$ $\cdots \rightarrow X_{m}$ with $m>0$ in $\mathbf{C}_{\mathbf{n}}(\operatorname{proj} \Lambda)$. If there are irreducible morphisms $f_{i}$ : $X_{i-1} \rightarrow X_{i}$ for $i \in\{1, \cdots, m\}$ such that $f_{m} \cdots f_{1} \in \mathfrak{R}_{\mathbf{C}_{\mathbf{n}}(\operatorname{proj} \Lambda)}^{m+1}\left(X_{0}, X_{m}\right), f_{m-1} \cdots f_{1} \notin$ $\Re_{\mathbf{C}_{\mathbf{n}(\operatorname{proj} \Lambda)}^{m}}\left(X_{0}, X_{m-1}\right)$ and $f_{m} \cdots f_{2} \notin \Re_{\mathbf{C}_{\mathbf{n}}(\operatorname{proj} \Lambda)}^{m}\left(X_{1}, X_{m}\right)$ then the following conditions hold.

(a) The complexes $X_{1}, \cdots, X_{m-1}$ are neither projective nor injective.

(b) $X_{0}$ is injective.

(c) $X_{m}$ is projective.

(d) If there is no positive integer s such that $X_{0}=J_{s}(P)$ and $X_{m}=J_{s}(Q)$, for $P$ and $Q$ indecomposable projective modules then $f_{m} \cdots f_{1}=0$.

Proof By hypothesis the sectional paths (1) $X_{0} \stackrel{f_{1}}{\rightarrow} X_{1} \stackrel{f_{2}}{\rightarrow} \ldots \stackrel{f_{m-1}}{\rightarrow} X_{m-1}$ and (2) $X_{1} \stackrel{f_{2}}{\rightarrow}$ $X_{2} \stackrel{f_{3}}{\rightarrow} \ldots \stackrel{f_{m}}{\rightarrow} X_{m}$ are non-zero.

(a). We consider two cases:

Case 1: $X_{0}$ or $X_{m}$ are projective-injective. Without loss of generality, we may assume that $X_{0}$ is projective-injective. Since the path (1) satisfies Lemma $6.9(a),(b)$ or $(c)$, $X_{m-1}$ is not projective-injective and $X_{0}$ is injective then we infer that satisfies $(a)$. Hence, $X_{1}, \cdots, X_{m-1}$ are not projective.

On the other hand, if the path (2) satisfies $(a)$, by Proposition 6.10 we have that $f_{m} \cdots f_{1} \notin \Re_{\mathbf{C}_{\mathbf{n}(\operatorname{proj} \Lambda)}^{m+1}}\left(X_{0}, X_{m}\right)$ a contradiction to the hypothesis. The path (2) satisfies (b), therefore $X_{1}, \cdots, X_{m-1}$ are neither projective nor injective.

Case 2: $X_{0}$ and $X_{m}$ are not projective-injective. If the path (1) satisfies Lemma 6.9 (a) then $X_{1}, \cdots, X_{m}$ are not projective. Hence, by Proposition 6.10 we have that $f_{m} \cdots f_{1} \notin$ $\Re_{\mathbf{C}_{\mathbf{n}}(\text { proj } \Lambda)}^{m+1}\left(X_{0}, X_{m}\right)$. If the path (2) satisfies $(b)$ then $X_{0}, \cdots, X_{m-1}$ are not injective. Therefore, by the dual of Proposition 6.10 we have that $f_{m} \cdots f_{1} \notin \Re_{\mathbf{C}_{\mathbf{n}}(\operatorname{proj} \Lambda)}^{m+1}\left(X_{0}, X_{m}\right)$. Clearly, the paths (1) and (2) satisfies only condition $(a)$ and $(b)$, respectively. Then, $X_{1}, \cdots, X_{m-1}$ are neither projective nor injective.

(b). Assume that $X_{0}$ is not injective. Since $(a)$ holds, by the dual of Proposition 6.10 we have that $f_{m} \cdots f_{1} \notin \Re_{\mathbf{C}_{\mathbf{n}}(\operatorname{proj} \Lambda)}^{m+1}\left(X_{0}, X_{m}\right)$. Therefore, $X_{0}$ is injective.

(c). If $X_{m}$ is not projective then by Proposition 6.10 we have that $f_{m} \cdots f_{1} \notin$ $\Re_{\mathbf{C}_{\mathbf{n}}(\operatorname{proj} \Lambda)}^{m+1}\left(X_{0}, X_{m}\right)$. Then, $X_{m}$ is projective.

$(d)$. Assume that $f_{m} \cdots f_{1} \neq 0$. By $(b)$ and $(c)$ we know that $X_{0}$ is injective and $X_{m}$ is projective. It is not hard to prove that there are not non-zero paths from $S(P)$ to $T(Q)$, 
from $S(P)$ to $J_{s}(Q)$ and from $J_{S}(P)$ to $T(Q)$. Then, $X_{0}=J_{s}(P)$ and $X_{m}=J_{t}(Q)$ with $t=s-1$ since $f_{m} \cdots f_{1} \neq 0$. Bellow, we illustrate the situation:
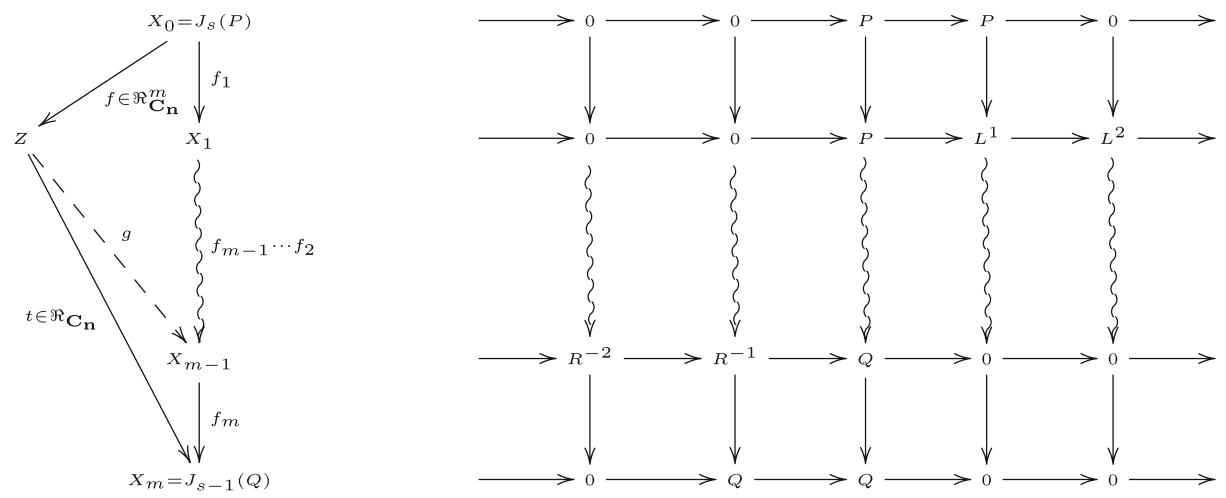

Consider $X_{0}=J_{s}(P), X_{m}=J_{s-1}(Q)$. Since $f_{m} \cdots f_{1} \in \Re_{\mathbf{C}_{\mathbf{n}}(\operatorname{proj} \Lambda)}^{m+1}\left(J_{s}(P), J_{s-1}(Q)\right)$ then $f_{m} \cdots f_{1}=t f$ with $t \in \Re_{\mathbf{C}_{\mathbf{n}}(\operatorname{proj} \Lambda)}\left(Z, J_{s-1}(Q)\right)$ and $f \in \mathfrak{R}_{\mathbf{C}_{\mathbf{n}}(\operatorname{proj} \Lambda)}^{m}\left(J_{s}(P), Z\right)$, for some complex $Z$. Moreover, $t$ does not split and $f_{m}$ is a minimal right almost split morphism. Hence, there is a morphism $g$ such that $t=f_{m} g$. Let $\gamma=g f \in$ $\Re_{\mathbf{C}_{\mathbf{n}}(\operatorname{proj} \Lambda)}^{m}\left(J_{S}(P), X_{m-1}\right)$. Then, $f_{m}\left(f_{m-1} \cdots f_{1}-\gamma\right)=0$.

On the other hand, because of the shape of $X_{0}$ and $X_{m}$ both morphisms $f_{m}\left(f_{m-1} \cdots f_{1}-\right.$ $\gamma$ ) and $f_{m-1} \cdots f_{1}-\gamma$ have their entries zero, for all entries different from $s$. More precisely, $f_{m}^{s}=i d_{Q}$. Then, $0=f_{m}^{s}\left(f_{m-1}^{s} \cdots f_{1}^{s}-\gamma^{s}\right)$ and we get that $f_{m-1} \cdots f_{1}=\gamma \in$ $\Re_{\mathbf{C}_{\mathbf{n}}(\operatorname{proj} \Lambda)}^{m}\left(X_{0}, X_{m-1}\right)$ a contradiction to the hypothesis. We conclude that $f_{m} \cdots f_{1}=0$.

In [9, Lemma 3.1], the authors omitted to analyzed the case where the complex $R(P)$ is not indecomposable, whenever $P$ is an indecomposable projective module. Even though, the statement of the result is true. For the convenience of the reader we complete the proof below.

Lemma 6.13 Let $\Lambda$ be an artin algebra and $X, Y, Z$ indecomposable complexes in $\mathbf{C}_{\mathbf{n}}(\operatorname{proj} \Lambda)$. If there are irreducible morphisms $h: X \rightarrow Y$ and $h^{\prime}: Y \rightarrow Z$ en $\mathbf{C}_{\mathbf{n}}(\operatorname{proj} \Lambda)$ such that $0 \neq h^{\prime} h \in \Re_{\mathbf{C}_{\mathbf{n}}(\operatorname{proj} \Lambda)}^{3}(X, Z)$ then $Z$ is not projective.

Proof Assume that $Z$ is projective. The idea of this proof is to show that under our assumption $X$ is an indecomposable injective and this fact will lead us to a contradiction.

Since $Z$ is an indecomposable projective then $Z=J_{j}(P)$ for $1 \leq j<n-1$ or $Z=$ $T(P)$ with $P$ an indecomposable projective module. By Theorem 4.2, there is a minimal right almost split morphism $\rho(P): R(P) \rightarrow Z$ in $\mathbf{C}_{\mathbf{n}}(\operatorname{proj} \Lambda)$.

In any case we have an exact sequence in $\mathbf{C}_{\mathbf{n}}(\bmod \Lambda)$ of the form

$$
0 \longrightarrow X_{1} \stackrel{\left(l, l^{\prime}\right)^{t}}{\longrightarrow} Y \oplus Y^{\prime} \stackrel{\left(h^{\prime}, k\right)}{\longrightarrow} Z
$$

where $R(P)=Y \oplus Y^{\prime}$. Observe that if $X_{1} \neq 0$ then it is a complex in $\mathbf{C}_{\mathbf{n}}(\bmod \Lambda)$.

On the other hand, $X_{1}$ admits a right $\mathbf{C}_{\mathbf{n}}(\operatorname{proj} \Lambda)$-approximation, $v: W \rightarrow X_{1}$, see [7, Theorem 4.5]. We will show that $X$ is an indecomposable injective direct summand of $W$. First, we prove that $W$ is a direct sum of indecomposable injective complexes, $W=$ $\oplus_{j=1}^{r-1} S\left(N_{j}\right) \oplus \oplus_{i=1}^{r-1} J_{i}\left(M^{i}\right)$. 
In order to compute the approximation, by [7, Theorem 4.5] we consider a quasiisomorphism $q: Q \rightarrow X_{1}$ with $Q \in \mathbf{C}^{\leq n}\left(\operatorname{proj} \Lambda\right.$ ) and the morphism $F(q): F(Q) \rightarrow X_{1}$. We have that $v=(F(q), \psi): F(Q) \oplus \oplus_{i=1}^{r-1} J_{i}\left(M^{i}\right) \rightarrow X_{1}$ is a right $\mathbf{C}_{\mathbf{n}}(\operatorname{proj} \Lambda)$ approximation, where $M^{i}$ is the projective cover of $X_{1}^{i}$.

To calculate $Q$ we have into account that $X_{1}$ has all its homologies being zero except for the first one, that we denote by $M$. Moreover, in the derived category we can identify $X_{1}$ with the shift of the module $M$.

In the case $Z=J_{j}(P)$ with $j \neq n$, it is enough to consider the projective resolution of $M=\operatorname{ker} d_{R}^{-j}$, that is, the exact sequence $\cdots \rightarrow R^{-j-2} \rightarrow R^{-j-1} \rightarrow 0 \rightarrow 0 \cdots$ in $\mathbf{C}_{\mathbf{n}}(\operatorname{proj} \Lambda)$. Then, $F(Q)$ is the complex $R^{-j-1} \rightarrow 0 \rightarrow 0 \cdots$ in $\mathbf{C}_{\mathbf{n}}(\operatorname{proj} \Lambda)$.

In case $Z=T(P)$, we consider $M=\operatorname{Ker} d_{R}^{-n}$ and $F(Q): R^{-n-1} \rightarrow 0 \rightarrow 0 \cdots$. Note that $F(Q)$ is an injective complex sum of indecomposable injective complexes of the form $S\left(N_{j}\right)$.

Now, we will concentrate on proving that $X$ is a direct summand of $W$. Since $h^{\prime} h \in$ $\mathfrak{R}_{\mathbf{C}_{\mathbf{n}}(\operatorname{proj} \Lambda)}^{3}(X, Z)$, there is a morphism $f^{\prime} \in \mathfrak{R}_{\mathbf{C}_{\mathbf{n}}(\operatorname{proj} \Lambda)}^{2}(X, N)$ and $f \in \mathfrak{R}_{\mathbf{C}_{\mathbf{n}}(\operatorname{proj} \Lambda)}(N, Z)$ such that $f f^{\prime}=h^{\prime} h$. Since $\left(h^{\prime}, k\right)$ is a right almost split morphism in $\mathbf{C}_{\mathbf{n}}(\operatorname{proj} \Lambda)$ and $f \in$ $\Re_{\mathbf{C}_{\mathbf{n}}(\operatorname{proj} \Lambda)}(N, Z)$ there is a morphism $\left(k_{1}, k_{2}\right)^{t}: N \rightarrow Y \oplus Y^{\prime}$ such that $\left(h^{\prime}, k\right)\left(k_{1}, k_{2}\right)^{t}=$ $f$. We write $(a, b)^{t}=\left(k_{1}, k_{2}\right)^{t} f^{\prime} \in \Re_{\mathbf{C}_{\mathbf{n}}(\operatorname{proj} \Lambda)}^{2}\left(X, Y \oplus Y^{\prime}\right)$. Then, $h^{\prime} h=\left(h^{\prime}, k\right)(a, b)^{t}=$ $h^{\prime} a+k b$. Hence, $\left(h^{\prime}, k\right)(a-h, b)^{t}=0$. Therefore, $(a-h, b)^{t}$ factorize through $X_{1}$. Then, there is $g^{\prime}: X \rightarrow X_{1}$ such that $l g^{\prime}=a-h$ and $l^{\prime} g^{\prime}=b$.

Since $v: W \rightarrow X_{1}$ is a right $\mathbf{C}_{\mathbf{n}}(\operatorname{proj} \Lambda)$-approximation of $X_{1}$, and there is a morphism $g: X \rightarrow W$ in $\mathbf{C}_{\mathbf{n}}\left(\operatorname{proj} \Lambda\right.$ ) such that $v g=g^{\prime}$. Then, $l^{\prime} v g=b$ and $a-h=l v g$. We illustrate the situation:

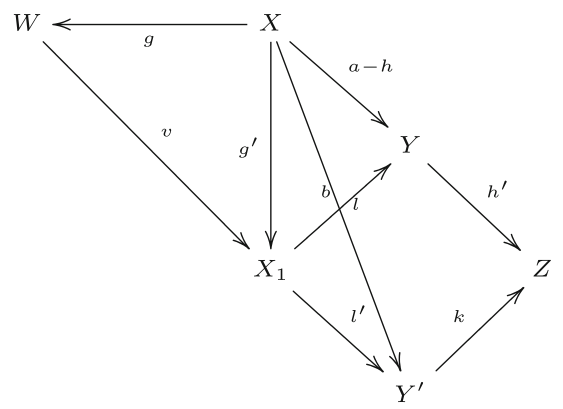

Assume that $g \in \Re_{\mathbf{C}_{\mathbf{n}}(\operatorname{proj} \Lambda)}(X, W)$, then $l v \notin \Re_{\mathbf{C}_{\mathbf{n}}(\operatorname{proj} \Lambda)}(W, Y)$ otherwise $h=l^{\prime} v g+$ $a \in \mathfrak{R}_{\mathbf{C}_{\mathbf{n}}(\operatorname{proj} \Lambda)}^{2}(X, Y)$, a contradiction to the fact that $h$ is irreducible. The morphism $l v$ is a retraction since $Y$ is indecomposable. Hence, $Y$ is a direct summand of $W$. Since $W$ is injective then $Y$ is an indecomposable injective. But this contradicts the fact that there are no irreducible morphisms from an indecomposable injective to an indecomposable projective. Then, $g \notin \Re_{\mathbf{C}_{\mathbf{n}}(\operatorname{proj} \Lambda)}(X, W)$. Therefore, $g$ is a section since $X$ is indecomposable. This proves that $X$ is injective.

Since there is a non-zero morphism from $X$ to $Z$, we get that $X \neq S(P)$. Moreover, if $X=J_{i}(P)$ then $Z \neq T(Q)$. Hence, $Z=J_{t}(Q)$ and $k=0, Y^{\prime}=0$ and $h^{\prime}(a-h)=$ 0 . Finally, by the shape of the minimal almost split morphisms if $X=J_{i}(P)$ and $h^{\prime} h$ : $J_{i}(P) \rightarrow J_{t}(Q)$ is a non-zero morphism then $i=t$. This leads us to a contradiction since $h^{\prime}(a-h)=0$ and $h^{\prime}(a-h): J_{i}(P) \rightarrow J_{i}(Q)$ is a composition of irreducible morphisms.

If $X_{1}=0$ the pair $\left(h^{\prime}, k\right)$ is a monomorphism and $0 \neq h h^{\prime} \in \mathfrak{R}_{\mathbf{C}_{\mathbf{n}}(\operatorname{proj} \Lambda)}^{3}(X, Z)$. As we explain before, $\left(h^{\prime}, k\right)(a-h, b)^{t}=0$ then $a-h=0$ and $h=a \in \mathfrak{i}_{\mathbf{C}_{\mathbf{n}}(\operatorname{proj} \Lambda)}^{2}(X, Y)$ is a contradiction since $h$ is irreducible. 
For a sectional path of length $m$ starting and ending in $J_{S}(P)$ and $J_{S}(Q)$, respectively, with $P$ and $Q$ indecomposable projective modules, we still do not have an answer to decide in which power of the radical the composition of the morphisms in such a path belongs. We have only an affirmative answer in case the length of the path is two. We expect to have a similar answer for any length.

Proposition 6.14 If $X_{0}=J_{s}(P) \rightarrow X_{1} \rightarrow \cdots \rightarrow X_{m-1} \rightarrow J_{s}(Q)=X_{m}$ is a path between indecomposable complexes in $\mathbf{C}_{\mathbf{n}}(\operatorname{proj} \Lambda)$ with $P, Q$ indecomposable projective $\Lambda$-modules and there are irreducible morphisms $f_{i}: X_{i-1} \rightarrow X_{i}$ for each $i \in\{1, \cdots, m\}$ then the following conditions hold.

(1) The path $J_{s}(P) \stackrel{f_{1}}{\rightarrow} X_{1} \stackrel{f_{2}}{\rightarrow} J_{s}(Q)$ is sectional, $f_{2} f_{1} \neq 0$ and $f_{2} f_{1} \notin$ $\Re_{\mathbf{C}_{\mathbf{n}}(\operatorname{proj} \Lambda)}^{3}\left(J_{S}(P), J_{S}(Q)\right)$.

(2) If $m=3$ then $J_{S}(P) \rightarrow X_{1} \rightarrow X_{2} \rightarrow J_{S}(Q)$ is a non-zero sectional path.

(3) If the path is sectional and the projective dimension of $Q / \mathrm{rad} Q$ is one then $f_{m} \cdots f_{1} \notin$ $\Re_{\mathbf{C}_{\mathbf{n}}(\operatorname{proj} \Lambda)}^{m+1}\left(J_{s}(P), J_{s}(Q)\right)$.

Proof (1). Assume that $f_{2} f_{1}=0$. Since $X_{1}=R_{S}(Q)=L_{S}(P)$ then $d=d_{Q}^{-1}=d_{L}^{1}=0$. We illustrate the situation with the following diagram
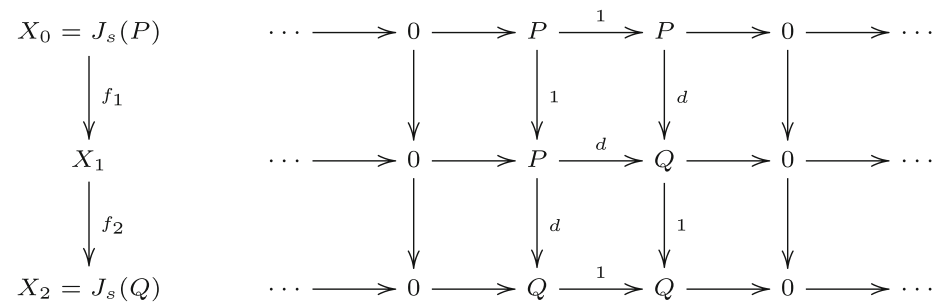

Hence, $X_{1}$ is not indecomposable which is a contradiction. Now, if $f_{2} f_{1} \in$ $\Re_{\mathbf{C}_{\mathbf{n}}(\operatorname{proj} \Lambda)}^{3}\left(X_{0}, X_{2}\right)$, since $f_{2} f_{1} \neq 0$ by [9, Lemma 3.1] we have that $X_{2}$ is not projective a contradiction to the fact that $X_{2}=J_{s}(Q)$.

(2). The complexes $J_{S}(P)$ and $J_{S}(Q)$ are projective-injective then clearly the path is sectional. To prove that it is non-zero, by (2.4) since $f_{2}$ is of the form ( $\mathrm{sec}$ ), ( $\mathrm{ret}$ ) or ( $\mathrm{ret}-$ irred $-s e c$ ) we analyze the different possibilities for $f_{2}$. We illustrate the situation with the following diagram

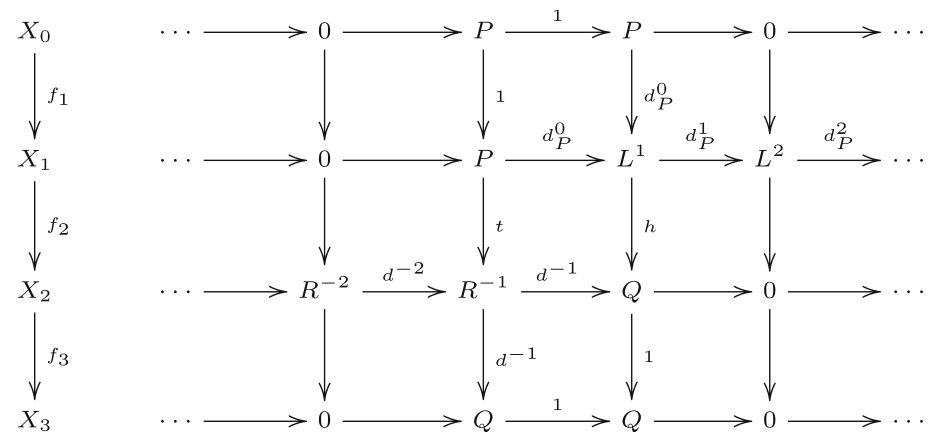

If $f_{2}$ is of the form ( $\mathrm{sec}$ ) then $L^{1}=Q, L^{j}=0$ if $j>1$. Therefore, $h$ is an isomorphism and $d^{-1} t=h d_{P}^{0}$ is irreducible, since $d_{P}^{0}$ is irreducible. Hence, $f_{3} f_{2} f_{1} \neq 0$. 
If $f_{2}$ is of the form ( $r e t$ ) then $R^{-1}=P$ and $R^{-i}=0$, for $i \geq 2$. Therefore, $t$ is an isomorphism. Hence, $d^{-1} t=h d_{P}^{0}$ and $d^{-1}$ is irreducible. Then, $f_{3} \bar{f}_{2} f_{1} \neq 0$.

Finally, we prove that $f_{2}$ is not of the form $($ ret-irred $-s e c)$. In fact, if $t$ is irreducible then $R^{-i}=0$ for $i \geq 2$. Moreover, $h$ is a section. Since $Q$ is indecomposable $Q=L^{1}$, that is, $h$ is an isomorphism. We get that $d^{-1} t \in \Re_{\mathbf{C}_{\mathbf{n}}(\operatorname{proj} \Lambda)}^{2}$ and $h d_{P}^{0} \in \Re_{\mathbf{C}_{\mathbf{n}}(\operatorname{proj} \Lambda)} \backslash \Re_{\mathbf{C}_{\mathbf{n}}(\operatorname{proj} \Lambda)}^{2}$ a contradiction since $d^{-1} t=h d_{P}^{0}$. Similarly, we can prove that $h$ is not irreducible.

(3). Assume that $f_{m} \cdots f_{1} \in \Re_{\mathbf{C}_{\mathbf{n}}(\operatorname{proj} \Lambda)}^{m+1}\left(X_{0}, X_{m}\right)$. Proceeding as in the proof of Theorem $6.12(d)$, considering the entries, we get the result with a similar analysis.

Acknowledgments The first and second authors thankfully acknowledge partial support from CONICET and from Universidad Nacional de Mar del Plata, Argentina. The third author thankfully acknowledge support from Ministerio Español de Economía y Competitividad and FEDER (FF12014-51978-C2-2-R). The first author is a researcher from CONICET.

\section{References}

1. Assem, I., Simson, D., Skowroński, A.: Elements of the representation theory of associative algebras. London Math. Soc. Student Texts 65. Cambridge University Press (2006)

2. Auslander, M., Reiten, I., Smalø, S.: Representation theory of artin algebras. vol 36 of Cambridge Studies in Advanced Mathematics. Cambridge University Press (1995)

3. Bautista, R.: Sections in Auslander-Reiten quivers. Representation theory II. In: Proceedings of the Second International Conference, Carleton University, Ottawa, Ont., 1979, Lecture Notes in Math. 832, pp. 74-96. Springer, Berlin-New York (1980)

4. Bautista, R.: The category of morphisms between projectives modules. Communications in Algebra 32(11), 4303-4331 (2004)

5. Bautista, R., Smalø, S.O.: Non-existent cycles. Communications in Algebra 11, 1755-1767 (1983)

6. Bautista, R., Souto Salorio, M.J.: Irreducible morphisms in the bounded derived category. Journal Pure and Applied 215, 866-884 (2011)

7. Bautista, R., Souto Salorio, M.J., Zuazua, R.: Almost split sequences for complexes of fixed size. Journal of Algebra 287, 140-168 (2005)

8. Chaio, C., Platzeck, M.I., Trepode, S.: On the degree of irreducible morphisms. Journal of Algebra 281 1, 200-224 (2004)

9. Chaio, C., Souto Salorio, M.J., Trepode, S.: Composite of irreducible morphisms in the bounded derived category. Journal of Pure and Applied Algebra 215(12), 2957-2968 (2011)

10. Girardo, H., Merklen, H.: Irreducible morphism of the category of complexes. Journal of Algebra 321, 2716-2736 (2009)

11. Happel, D.: Triangulated categories in the representation theory of finite dimensional algebras. London Math. Soc. Lecture Note Ser., vol. 119, Cambridge (1998)

12. Igusa, K., Todorov, G.: A characterization of finite Auslander-Reiten quivers. Journal Algebra 89, 148177 (1984)

13. Liu, S.: Auslander-reiten theory in a Krull-Schmidt category. Sao Paulo J. Math. Sci. 4, 425-472 (2010)

14. Wheeler, W.: The triangulated structure of the stable derived category. Journal of Algebra 165(1), 23-40 (1994) 